\title{
Thermal [3+2] Cycloaddition of Aromatic Azomethine Imines with Allenoates
}

Lei Zhang, Chengfeng Jing, Honglei Liu, Bo Wang, Zhen Li, Hui Jiang, Hao Yu, Hongchao Guo*

Department of Applied Chemistry, China Agricultural University, Beijing 100193, P. R. of China

Fax+86(10)62820325; E-mail: hchguo@cau.edu.cn

Received: 05.10.2012; Accepted after revision: 09.11.2012

\begin{abstract}
The thermal [3+2] cycloadditions of two classes of aromatic azomethine imines with allenoates have been investigated. The reactions are operationally simple and proceed smoothly under mild reaction conditions to provide a variety of dinitrogen-fused heterocycles in moderate to excellent yields.
\end{abstract}

Key words: cycloaddition, azomethine imine, allenoate, quinoline, isoquinoline

Quinoline, isoquinoline and structurally related heterocycles occur widely in nature and some have a broad range of clinical applications, exhibiting a wide range of biological activities such as antitumor, anti-HIV, antibiotic, antifungal, antivirus, anti-inflammatory, anticoagulation, and bronchodilation, and can also act on the central nervous system. ${ }^{1}$ For example, Ecteinascidin 743 (Yondelis, trabectedin; Figure 1) is a new antitumor agent of marine origin discovered in the Caribbean tunicate Ecteinascidia turbinate for the treatment of soft tissue sarcoma and ovarian cancer. ${ }^{2}$ Phase II trials with Yondelis are also being carried out for breast cancer, lung cancer, prostate cancer, and for paediatric tumors. Higenamine ${ }^{3}$ and RESPIR 4$95^{4}$ (Figure 1) display anticoagulation and bronchodilation activities, respectively. Compounds I (Figure 1) are potent nonnucleoside, allosteric inhibitors of reverse transcriptase, and could interact with retroviral targets relevant to anti-HIV therapy. ${ }^{5}$ The tetrahydroquinoline derivative II shows activity against antibacterial targets such as DNA gyrase. ${ }^{6}$ Accordingly, the synthesis of quinoline and isoquinoline derivatives has attracted much attention. $^{7}$ However, although many synthetic methods have been developed, ${ }^{7}$ new procedures for their synthesis would still be highly desirable.

Azomethine imines have emerged as a versatile 1,3-dipole for various thermal, metal-catalyzed, and organocatalytic 1,3-dipolar cycloaddition reactions and have been extensively applied in organic synthesis because of their easy accessibility, stability and the potential applications of the corresponding cycloadducts. ${ }^{8,9}$ The range of azomethine imines available for the cycloaddition reactions has been expanded to include various imines such as 3-oxopyrazolidin-1-ium-2-ide derivatives and benzoyl(3,4-dihydroisoquinolin-2-ium-2-yl)amides, but research on aromatic azomethine imines is somewhat limited. ${ }^{\text {z, }, 10} \mathrm{Re}-$ cently, we successfully developed [3+2], [3+3], [4+3],

SYNTHESIS 2013, 45, 0053-0064

Advanced online publication: 06.12.2012

DOI: 10.1055/s-0032-1316822; Art ID: SS-2012-H0773-OP

(C) Georg Thieme Verlag Stuttgart $\cdot$ New York and $[3+2+3]$ annulation reactions of azomethine imines with allenoates, affording facile access to dinitrogenfused bicyclic or tricyclic heterocycles, ${ }^{11}$ which are key units in or building blocks of many pharmaceuticals, agrochemicals, biologically active compounds, and other useful chemicals. ${ }^{12}$ As part of our continuing efforts to develop the annulation reaction of azomethine imines, ${ }^{11}$ we recently investigated the reactions of aromatic azomethine imines with allenoates. Herein, we present our results on the thermal [3+2] cycloaddition of aromatic azomethine imines with allenoates to furnish functionalized dinitrogen-fused tricyclic heterocycles.

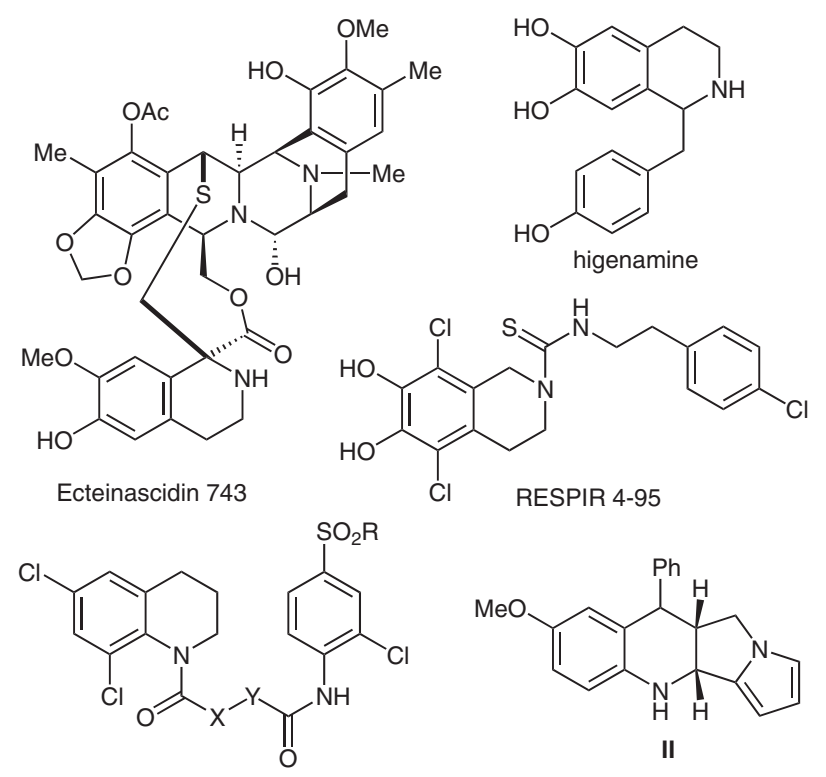

I, $\mathrm{X}-\mathrm{Y}=\mathrm{SCH}_{2}, \mathrm{OCH}_{2}, \mathrm{NHCH}_{2}$, etc.; $\mathrm{R}=\mathrm{NH}_{2}, \mathrm{Me}$

Figure 1 Pharmaceutically active quinoline and isoquinoline-related derivatives

The azomethine imines $\mathbf{1 ,} \mathbf{8}$ and $\mathbf{1 3}$ were easily prepared according to reported procedures. ${ }^{13}$ In initial attempts, azomethine imine $\mathbf{1}$ was treated with the allenoate $\mathbf{2 a}$ in dichloromethane at room temperature for 96 hours (Scheme 1). The reaction worked sluggishly, and two new products were isolated in total $20 \%$ yield. When the reaction was carried out in toluene at room temperature for 96 hours, the same two products were obtained in poor yield $(5 \%)$ due to the poor solubility of azomethine imine 1 in toluene. To increase the yield, the reaction was carried out in refluxing toluene. Under these conditions the azomethine imine was completely converted in 72 hours to give the product in $70 \%$ yield. However, several side- 
products were observed in small amounts. Decreasing the reaction temperature to $90{ }^{\circ} \mathrm{C}$ resulted in a clean reaction and improved yield ( $89 \%)$. The two products could be separated by silica gel chromatography, and were determined to be [3+2] cycloaddition products $3 \mathbf{a}$ and $4 \mathbf{a}$ (Scheme 1), on the basis of 2D NMR spectroscopic and $\mathrm{X}$-ray crystallographic ${ }^{14}$ analyses (Figure 2). In principle, since both active double bonds in allenoate could undergo the cycloaddition reaction with azomethine imine, three kinds of cycloadducts could be produced in the reaction, but the reaction was highly regioselective and produced the 2-exo methylene isomer as the sole regioisomer, which possessed two chiral carbons (Scheme 1). It is noteworthy that an interesting quaternary carbon center was incorporated into the molecular skeleton of the products $\mathbf{3 a}$ and $\mathbf{4 a}$.

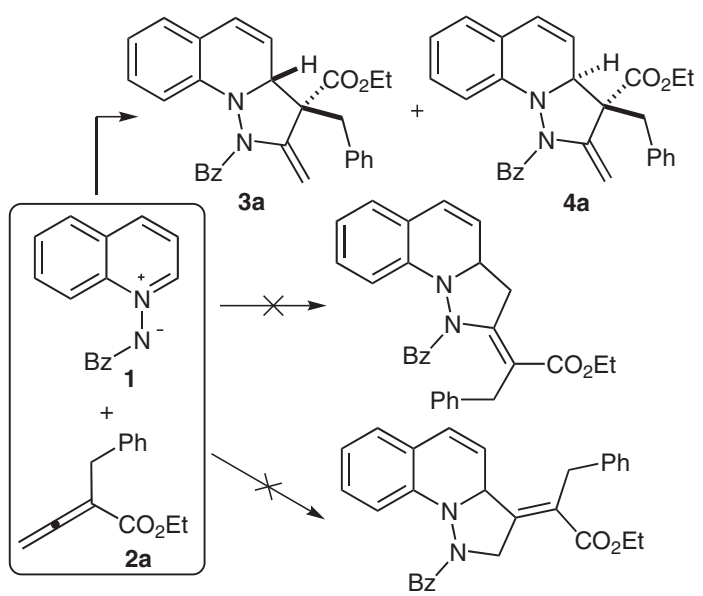

Scheme 1 The thermal cycloaddition of azomethine imine 1 with ethyl $\alpha$-substituted allenoate $\mathbf{2 a}$

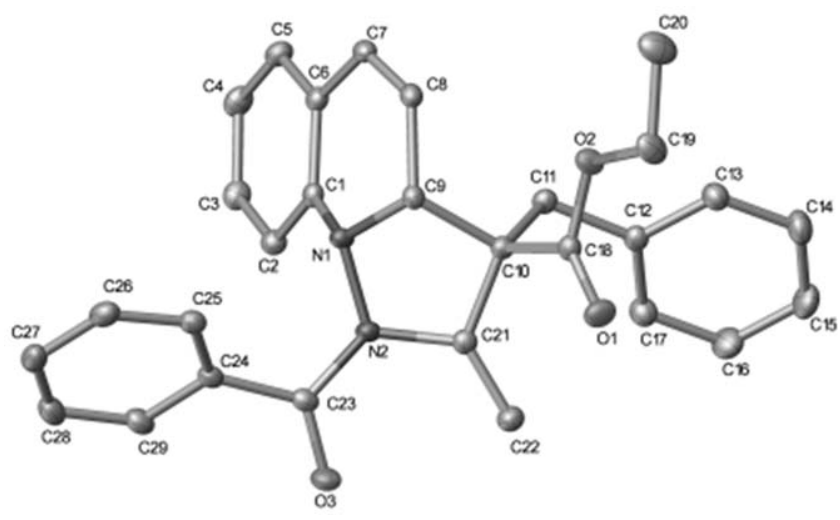

Figure 2 The X-ray crystal structure of $\mathbf{4 a}$

With the optimal reaction conditions in hand (toluene, $90{ }^{\circ} \mathrm{C}$ ), azomethine imine 1 was treated with various substituted allenoates 2 in toluene for 72 hours, giving the functionalized dinitrogen-fused tricyclic heterocycles $\mathbf{3}$ and $\mathbf{4}$ in moderate to excellent yields, albeit with poor diastereoselectivity (Table 1, entries 1-13). Although poor
Table 1 Thermal Cycloaddition of Azomethine Imine 1 with $\alpha$-Substituted Allenoates $\mathbf{2}^{\mathrm{a}}$

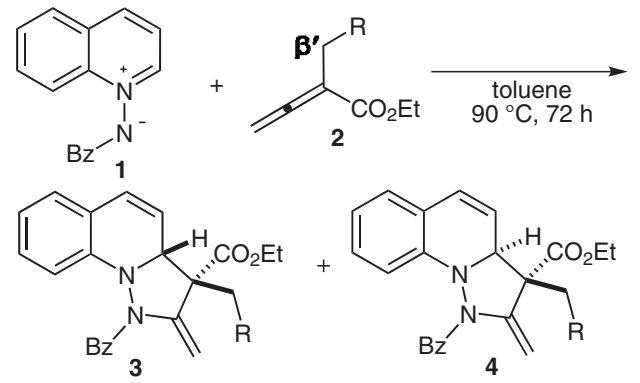

\begin{tabular}{lllll}
\hline Entry & $\mathrm{R}$ & Product & Yield (\%) $^{\mathrm{b}}$ & Ratio $\mathbf{3} / \mathbf{4}^{\mathbf{c}}$ \\
\hline 1 & $\mathrm{Ph}(\mathbf{2 a})$ & $\mathbf{3 a}+\mathbf{4 a}$ & 89 & $53: 47$ \\
2 & $2-\mathrm{MeC}_{6} \mathrm{H}_{4}(\mathbf{2 b})$ & $\mathbf{3 b}+\mathbf{4 b}$ & 96 & $41: 59$ \\
3 & $3-\mathrm{MeC}_{6} \mathrm{H}_{4}(\mathbf{2 c})$ & $\mathbf{3 c}+\mathbf{4 c}$ & 81 & $52: 48$ \\
4 & $4-\mathrm{MeC}_{6} \mathrm{H}_{4}(\mathbf{2 d})$ & $\mathbf{3 d}+\mathbf{4 d}$ & 79 & $57: 43$ \\
5 & $2-\mathrm{FC}_{6} \mathrm{H}_{4}(\mathbf{2 e})$ & $\mathbf{3 e}+\mathbf{4 e}$ & 94 & $49: 51$ \\
6 & $3-\mathrm{FC}_{6} \mathrm{H}_{4}(\mathbf{2} \mathbf{f})$ & $\mathbf{3 f}+\mathbf{4 f}$ & 86 & $57: 43$ \\
7 & $4-\mathrm{FC}_{6} \mathrm{H}_{4}(\mathbf{2 g})$ & $\mathbf{3 g}+\mathbf{4 g}$ & 82 & $52: 48$ \\
8 & $2-\mathrm{ClC}_{6} \mathrm{H}_{4}(\mathbf{2 h})$ & $\mathbf{3 h}+\mathbf{4 h}$ & 91 & $40: 60$ \\
9 & $3-\mathrm{ClC}_{6} \mathrm{H}_{4}(\mathbf{2 i})$ & $\mathbf{3 i}+\mathbf{4 i}$ & 68 & $40: 60$ \\
10 & $4-\mathrm{ClC}_{6} \mathrm{H}_{4}(\mathbf{2 j})$ & $\mathbf{3 j}+\mathbf{4 j}$ & 65 & $60: 40$ \\
11 & $2-\mathrm{BrC}_{6} \mathrm{H}_{4}(\mathbf{2 k})$ & $\mathbf{3 k}+\mathbf{4 k}$ & 73 & $45: 55$ \\
12 & $3-\mathrm{BrC}_{6} \mathrm{H}_{4}(\mathbf{2 l})$ & $\mathbf{3 i}+\mathbf{4 l}$ & 70 & $53: 47$ \\
13 & $\left.\mathrm{CO}_{2} \mathrm{Et}_{(\mathbf{2}} \mathbf{2 m}\right)$ & $\mathbf{3 m}+\mathbf{4 m}$ & 86 & $74: 26^{\mathrm{d}}$ \\
\hline
\end{tabular}

a 1.2 equivalent of allenoate was used.

${ }^{\mathrm{b}}$ Isolated yields.

${ }^{\mathrm{c}}$ Based on isolated yield unless otherwise stated.

${ }^{\mathrm{d}}$ Based on integration of signals in the ${ }^{1} \mathrm{H}$ NMR spectrum.

diastereoselectivities were obtained, both diastereomers could be separated, providing diverse heterocycles for medicinal chemistry. The allenoates with electron-withdrawing substituents in the aryl group worked as well as those allenoates with electron-donating substituents in the aryl group under otherwise identical conditions (entries 1-12), and the derivatives of ethyl 1-benzoyl-2-methylene-1,2,3,3a-tetrahydropyrazolo[1,5-a]quinoline-3-carboxylate could be obtained in $65-96 \%$ yield. The highest yield $(96 \%)$ was achieved in the cycloaddition of $\beta^{\prime}-2$ $\mathrm{MeC}_{6} \mathrm{H}_{4}$ substituted allenoate (entry 2). Although the $\mathrm{R}$ substituent in allenoate is some distance away from the active double bond, it seems that the position of $\mathrm{R}$ relative to the $\beta^{\prime}$-position of allenoate has an influence on the yield. In general, in comparison with allenoates with meta- and para-substituents in the aryl group, allenoates with ortho-substituents in the aryl group underwent the reaction in higher yields (entries 2, 5, 8, and 11). The $\beta^{\prime}$ ethoxycarbonyl allenoate was active and smoothly partic- 
ipated in the cycloaddition to give the expected cycloadduct in $86 \%$ yield (entry 13), and ethyl buta-2,3-dienoate (5a) also worked efficiently to give the cycloadducts in $85 \%$ yield (Scheme 2). Unfortunately, the reaction with $\gamma$ substituted allenoate $\mathbf{5 b}$ was more sluggish and afforded the cycloadduct in moderate yield (Scheme 2).

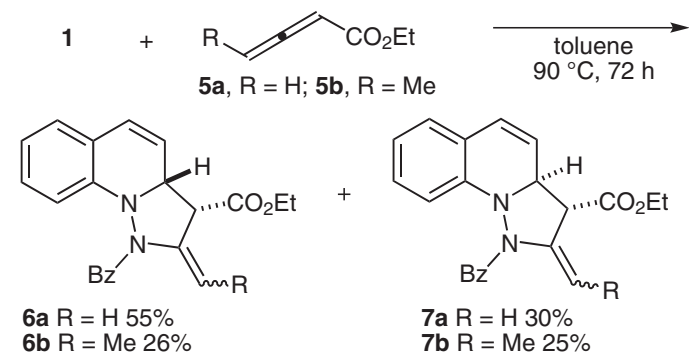

Scheme 2 Thermal cycloaddition of azomethine imine 1 with ethyl buta-2,3-dienoate (5a) and $\gamma$-substituted allenoate (5b)

Table 2 Thermal Cycloaddition of Azomethine Imine 8 with $\alpha$-Substituted Allenoates $\mathbf{2}^{\mathrm{a}}$

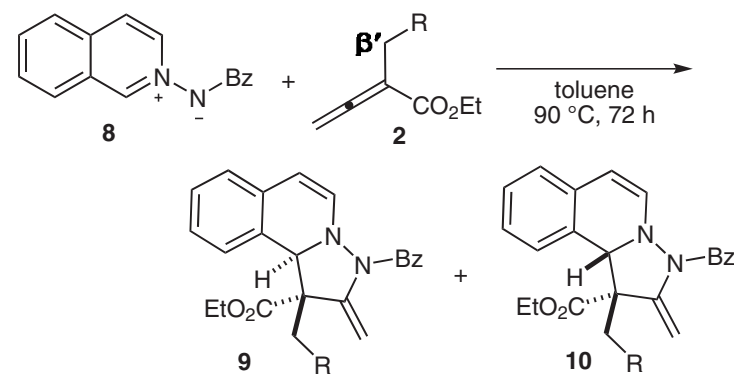

\begin{tabular}{lllll}
\hline Entry & $\mathrm{R}$ & Product & Yield (\%) $^{\mathrm{b}}$ & Ratio 9/10 \\
\hline 1 & $\mathrm{Ph}(\mathbf{2 a})$ & $\mathbf{9 a}+\mathbf{1 0 a}$ & 71 & $41: 59$ \\
2 & $2-\mathrm{MeC}_{6} \mathrm{H}_{4}(\mathbf{2 b})$ & $\mathbf{9 b}+\mathbf{1 0 b}$ & 50 & $72: 28^{\mathrm{d}}$ \\
3 & $3-\mathrm{MeC}_{6} \mathrm{H}_{4}(\mathbf{2 c})$ & $\mathbf{9 c}+\mathbf{1 0 c}$ & 75 & $40: 60^{\mathrm{d}}$ \\
4 & $2-\mathrm{FC}_{6} \mathrm{H}_{4}(\mathbf{2 e})$ & $\mathbf{9 e}+\mathbf{1 0 e}$ & 98 & $52: 48$ \\
5 & $3-\mathrm{FC}_{6} \mathrm{H}_{4}(\mathbf{2 f})$ & $\mathbf{9 f}+\mathbf{1 0 f}$ & 61 & $23: 77$ \\
6 & $4-\mathrm{FC}_{6} \mathrm{H}_{4}(\mathbf{2 g})$ & $\mathbf{9 g}+\mathbf{1 0 g}$ & 71 & $32: 68$ \\
7 & $2-\mathrm{ClC}_{6} \mathrm{H}_{4}(\mathbf{2 h})$ & $\mathbf{9 h}+\mathbf{1 0 h}$ & 72 & $51: 49$ \\
8 & $3-\mathrm{ClC}_{6} \mathrm{H}_{4}(\mathbf{2 i})$ & $\mathbf{9 i}+\mathbf{1 0 i}$ & 66 & $35: 65$ \\
9 & $3-\mathrm{BrC}_{6} \mathrm{H}_{4}(\mathbf{2 l})$ & $\mathbf{9 1}+\mathbf{1 0 1}$ & 76 & $41: 59$ \\
10 & $\left.\mathrm{CO}_{2} \mathrm{Et}_{\mathbf{2}} \mathbf{2 m}\right)$ & $\mathbf{9 m}+\mathbf{1 0 m}$ & 86 & $37: 63$ \\
\hline
\end{tabular}

a 1.2 equivalent of allenoate was used.

${ }^{\mathrm{b}}$ Isolated yield.

${ }^{\mathrm{c}}$ Based on isolated yield unless otherwise stated.

${ }^{\mathrm{d}}$ Based on integration of signals in the ${ }^{1} \mathrm{H}$ NMR spectrum.

Having investigated the reactions of aromatic azomethine imine $\mathbf{1}$, based on the dihydroquinoline structure, with allenoates, we then explored the reaction of aromatic azomethine imine 8, based on dihydroisoquinoline structure, with allenoates (Table 2). We were pleased to find that imine 8 was also active. A wide range of aryl groups with electron-donating or withdrawing substituents at the $\beta^{\prime}$ position of the allenoate could be tolerated in the cycloaddition. The reactions were carried out in toluene at $90{ }^{\circ} \mathrm{C}$ for 72 hours to afford derivatives of ethyl 3-benzoyl-2methylene-1,2,3,10b-tetrahydropyrazolo[5,1-a]isoquinoline-1-carboxylate in 50-98\% yields with moderate diastereoselectivities (entries 1-10). Fortunately, most diastereomeric mixtures could be separated by using flash column chromatography. However, the diastereomeric mixtures obtained from the reaction of imine $\mathbf{8}$ with either allenoate $\mathbf{2 b}$ or $\mathbf{2}$ c could not be separated by flash column chromatography, and the diastereomeric ratios were determined by NMR spectroscopic analysis. In contrast to reactions with azomethine imine 1 , the influence of the position of the $\mathrm{R}$ substituent relative to the $\beta^{\prime}$-position of the allenoate on the yields were not clear (entries 2, 3 vs 4-8). Ethyl buta-2,3-dienoate (5a) also smoothly underwent the cycloaddition reactions, affording the corresponding cycloadduct in good yield (Scheme 3). Azomethine imine 13, which is an analogue of 8, underwent the reaction to produce the desired product in $68 \%$ yield with a diastereomeric ratio of around 2:1 (Scheme 4).

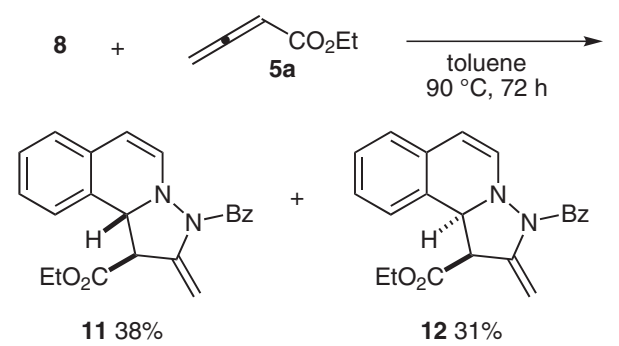

Scheme 3 Thermal cycloaddition of azomethine imine 8 with ethyl buta-2,3-dienoate (5a)

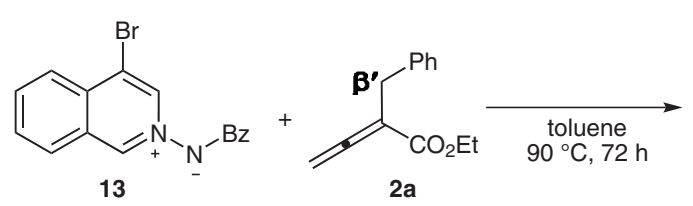

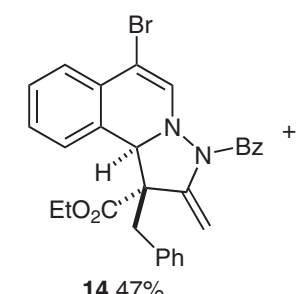<smiles>C=C1N2C=C(Br)c3ccccc3[C@@H]2[C@@H](CC(C)(C)C)N1Cc1ccccc1</smiles>

Scheme 4 Thermal cycloaddition of azomethine imine 13 with ethyl 2-benzylbuta-2,3-dienoate (2a)

In summary, thermal $[3+2]$ cycloaddition reactions of aromatic azomethine imines with allenoates have been developed; the reactions were performed in toluene at $90{ }^{\circ} \mathrm{C}$ to give the functionalized dinitrogen-fused tricyclic heterocycles, which are pharmaceutically important compounds. The desired products were obtained in moderate to excellent yields. The reaction is operationally simple 
and works efficiently, thus it is a potentially useful protocol for the synthesis of biologically active molecules.

Unless otherwise stated, all reagents were purchased from commercial suppliers and used without further purification. Organic solutions were concentrated under reduced pressure using a rotary evaporator or oil pump. Reactions were monitored by thin-layer chromatography (TLC) on silica gel precoated glass plates; chromatograms were visualized by fluorescence quenching under UV light at $254 \mathrm{~nm}$. Flash column chromatography was performed with Qingdao Haiyang flash silica gel (200-300 mesh). Infrared spectra were recorded with a Bruker Optics TENSOR 27 instrument. ${ }^{1} \mathrm{H}$ and ${ }^{13} \mathrm{C}$ NMR spectra were recorded with a Bruker-300 spectrometer. Accurate mass measurements were performed with an Agilent instrument using the ESI-MS technique. X-ray crystallographic data were collected with a Bruker SMART CCD-based diffractometer equipped with a low-temperature apparatus operated at $100 \mathrm{~K}$.

\section{[3+2] Annulation of Azomethine Imines with Allenoates; Gen-} eral Procedure

A mixture of azomethine imine $(0.125 \mathrm{mmol})$ and allenoate $(0.15$ $\mathrm{mmol})$ in toluene $(5 \mathrm{~mL})$ was stirred at $90^{\circ} \mathrm{C}$ for $72 \mathrm{~h}$ and then concentrated. The residue was purified by flash column chromatography (EtOAc-hexane) to afford the corresponding product. Some annulation products $(3 \mathrm{~m}$ and $4 \mathrm{~m}$ in Table $1 ; 9 \mathrm{~b}$ and $10 \mathrm{~b}, 9 \mathrm{c}$ and $10 \mathrm{c}$ in Table 2) were obtained as diastereomeric mixtures that could not be separated by flash silica gel column chromatography; in these cases the NMR spectra, the IR data, and HRMS data were collected for the diastereomeric mixture. Melting points are reported for crystalline solids but were not measured for amorphous solids.

\section{Compound 3a}

Yield: $26.7 \mathrm{mg}$ (47\%); white crystalline solid; mp 132.6-134.5 ${ }^{\circ} \mathrm{C}$. IR (film): 2979, 1722, 1666, 1599, 1484, 1454, 1339, 1292, 1261, $1222,1187,1095,1079,1043,1009,861,773,756,738,699,653$, $628 \mathrm{~cm}^{-1}$.

${ }^{1} \mathrm{H}$ NMR $\left(300 \mathrm{MHz}, \mathrm{CDCl}_{3}\right): \delta=7.39-7.26(\mathrm{~m}, 8 \mathrm{H}), 7.20-7.18(\mathrm{~m}$, $2 \mathrm{H}), 7.00-6.90(\mathrm{~m}, 1 \mathrm{H}), 6.78-6.70(\mathrm{~m}, 1 \mathrm{H}), 6.68-6.62(\mathrm{~m}, 2 \mathrm{H})$, $6.32(\mathrm{~d}, J=9.8 \mathrm{~Hz}, 1 \mathrm{H}), 6.06(\mathrm{~d}, J=1.4 \mathrm{~Hz}, 1 \mathrm{H}), 5.42-5.40(\mathrm{~m}$, $1 \mathrm{H}), 5.01(\mathrm{~d}, J=1.5 \mathrm{~Hz}, 1 \mathrm{H}), 4.45-4.43(\mathrm{~m}, 1 \mathrm{H}), 3.88-3.83(\mathrm{~m}$, $1 \mathrm{H}), 3.57-3.35(\mathrm{~m}, 2 \mathrm{H}), 3.24(\mathrm{~d}, J=14.3 \mathrm{~Hz}, 1 \mathrm{H}), 0.60(\mathrm{t}$, $J=7.2 \mathrm{~Hz}, 3 \mathrm{H})$.

${ }^{13} \mathrm{C} \mathrm{NMR}\left(75 \mathrm{MHz}, \mathrm{CDCl}_{3}\right): \delta=172.3,169.7,143.8,143.7,135.8$, $134.6,130.8,130.6,129.5,128.5,127.7,127.6,127.40,127.36$, $126.8,121.2,120.9,118.7,111.8,100.6,64.6,62.6,61.3,40.3$, 12.8 .

HRMS (ESI): $m / z[\mathrm{M}+\mathrm{H}]^{+}$calcd for $\mathrm{C}_{29} \mathrm{H}_{27} \mathrm{~N}_{2} \mathrm{O}_{3}{ }^{+}: 451.2016$; found: 451.2017 .

\section{Compound 3b}

Yield: $22.83 \mathrm{mg}$ (39\%); white crystalline solid; $\mathrm{mp} 164.2-165.6{ }^{\circ} \mathrm{C}$. IR (film): 3122, 3031, 2963, 2934, 1720, 1673, 1645, 1599, 1578 , $1485,1456,1447,1403,1368,1335,1294,1263,1233,1217,1191$, $1179,1159,1109,1088,1074,1018,956,931,914,883,863,835$, $795,779,758,726,702,661,631,601,562,531,520,462 \mathrm{~cm}^{-1}$.

${ }^{1} \mathrm{H}$ NMR $\left(300 \mathrm{MHz}, \mathrm{CDCl}_{3}\right): \delta=7.55-7.43(\mathrm{~m}, 1 \mathrm{H}), 7.36-6.97(\mathrm{~m}$, $9 \mathrm{H}), 6.79-6.77(\mathrm{~m}, 1 \mathrm{H}), 6.76-6.58(\mathrm{~m}, 2 \mathrm{H}), 6.33(\mathrm{~d}, J=10.0 \mathrm{~Hz}$ $1 \mathrm{H}), 6.05(\mathrm{~d}, J=1.4 \mathrm{~Hz}, 1 \mathrm{H}), 5.56-5.53(\mathrm{~m}, 1 \mathrm{H}), 5.03(\mathrm{~d}$, $J=1.5 \mathrm{~Hz}, 1 \mathrm{H}), 4.29-4.27(\mathrm{~m}, 1 \mathrm{H}), 3.91-3.86(\mathrm{~m}, 1 \mathrm{H}), 3.68(\mathrm{~d}$, $J=14.7 \mathrm{~Hz}, 1 \mathrm{H}), 3.49-3.46(\mathrm{~m}, 1 \mathrm{H}), 3.22(\mathrm{~d}, J=14.7 \mathrm{~Hz}, 1 \mathrm{H})$, $2.39(\mathrm{~s}, 3 \mathrm{H}), 0.60(\mathrm{t}, J=7.2 \mathrm{~Hz}, 3 \mathrm{H})$.

${ }^{13} \mathrm{C}$ NMR $\left(75 \mathrm{MHz}, \mathrm{CDCl}_{3}\right): \delta=172.6,169.6,144.1,143.8,137.4$, $134.6,134.3,130.54,130.51,130.3,129.6,127.7,127.6,127.3$, $127.2,126.8,126.4,121.2,120.9,119.2,111.8,101.2,65.2,62.7$, $61.3,35.9,20.5,12.8$.
HRMS (ESI): $m / z[\mathrm{M}+\mathrm{H}]^{+}$calcd for $\mathrm{C}_{30} \mathrm{H}_{29} \mathrm{~N}_{2} \mathrm{O}_{3}{ }^{+}$: 465.2173; found: 465.2184 .

\section{Compound 3c}

Yield: $24.2 \mathrm{mg}$ (42\%); white solid.

IR (film): 3025, 2978, 2924, 1727, 1671, 1647, 1600, 1578, 1485, $1455,1446,1403,1368,1344,1294,1262,1224,1188,1160,1096$, $1049,1026,1016,862,794,785,771,751 \mathrm{~cm}^{-1}$.

${ }^{1} \mathrm{H} \mathrm{NMR}\left(300 \mathrm{MHz}, \mathrm{CDCl}_{3}\right): \delta=7.37-7.26(\mathrm{~m}, 3 \mathrm{H}), 7.19-7.13(\mathrm{~m}$, $5 \mathrm{H}), 7.12-6.93(\mathrm{~m}, 2 \mathrm{H}), 6.78-6.75(\mathrm{~m}, 1 \mathrm{H}), 6.74-6.57(\mathrm{~m}, 2 \mathrm{H})$, $6.32(\mathrm{~d}, J=10.0 \mathrm{~Hz}, 1 \mathrm{H}), 6.03(\mathrm{~d}, J=11.9 \mathrm{~Hz}, 1 \mathrm{H}), 5.45-5.42(\mathrm{~m}$, $1 \mathrm{H}), 5.03(\mathrm{~d}, J=1.5 \mathrm{~Hz}, 1 \mathrm{H}), 4.45-4.43(\mathrm{~m}, 1 \mathrm{H}), 3.89-3.85(\mathrm{~m}$, $1 \mathrm{H}), 3.56-3.35(\mathrm{~m}, 2 \mathrm{H}), 3.21(\mathrm{~d}, J=14.3 \mathrm{~Hz}, 1 \mathrm{H}), 2.30(\mathrm{~s}, 3 \mathrm{H})$, $0.60(\mathrm{t}, J=7.2 \mathrm{~Hz}, 3 \mathrm{H})$.

${ }^{13} \mathrm{C}$ NMR $\left(75 \mathrm{MHz}, \mathrm{CDCl}_{3}\right): \delta=172.3,169.7,143.8,138.2,135.7$, $134.7,131.3,130.5,129.5,128.4,128.1,127.8,127.7,127.6,127.3$, $126.7,121.2,120.8,118.8,111.8,100.5,64.5,62.5,61.3,40.2$, $21.3,12.8$

HRMS (ESI): $m / z[\mathrm{M}+\mathrm{H}]^{+}$calcd for $\mathrm{C}_{30} \mathrm{H}_{29} \mathrm{~N}_{2} \mathrm{O}_{3}{ }^{+}: 465.2173$; found: 465.2174 .

\section{Compound 3d}

Yield: $26 \mathrm{mg}(45 \%)$; white crystalline solid; $\mathrm{mp} 163.4-165.4{ }^{\circ} \mathrm{C}$.

IR (film): 3064, 3029, 2960, 2923, 1721, 1671, 1645, 1601, 1516, $1484,1448,1405,1374,1348,1293,1263,1228,1187,1112,1093$, $1048,1025,1009,874,790,772,753,694 \mathrm{~cm}^{-1}$.

${ }^{1} \mathrm{H}$ NMR (300 MHz, $\left.\mathrm{CDCl}_{3}\right): \delta=7.39-7.15(\mathrm{~m}, 7 \mathrm{H}), 7.10(\mathrm{~d}$, $J=7.9 \mathrm{~Hz}, 2 \mathrm{H}), 7.00(\mathrm{~m}, 1 \mathrm{H}), 6.78-6.76(\mathrm{~m}, 1 \mathrm{H}), 6.67-6.63(\mathrm{~m}$, $2 \mathrm{H}), 6.31(\mathrm{~d}, J=10.0 \mathrm{~Hz}, 1 \mathrm{H}), 6.13-5.95(\mathrm{~m}, 1 \mathrm{H}), 5.42-5.39(\mathrm{~m}$, $1 \mathrm{H}), 5.01(\mathrm{~d}, J=4.2 \mathrm{~Hz}, 1 \mathrm{H}), 4.46-4.45(\mathrm{~m}, 1 \mathrm{H}), 3.88-3.82(\mathrm{~m}$, $1 \mathrm{H}), 3.59-3.34(\mathrm{~m}, 2 \mathrm{H}), 3.20(\mathrm{~d}, J=14.2 \mathrm{~Hz}, 1 \mathrm{H}), 2.30(\mathrm{~s}, 3 \mathrm{H})$, $0.60(\mathrm{t}, J=7.2 \mathrm{~Hz}, 3 \mathrm{H})$.

${ }^{13} \mathrm{C}$ NMR $\left(75 \mathrm{MHz}, \mathrm{CDCl}_{3}\right): \delta=172.3,169.7,143.8,143.8,136.9$, $134.7,132.6,130.62,130.57,129.5,129.1,127.7,127.6,127.4$, $126.7,121.2,120.8,118.8,111.8,100.4,64.6,62.6,61.2,39.9$, $21.0,12.8$

HRMS (ESI): $m / z[\mathrm{M}+\mathrm{H}]^{+}$calcd for $\mathrm{C}_{30} \mathrm{H}_{29} \mathrm{~N}_{2} \mathrm{O}_{3}{ }^{+}: 465.2173$; found: 465.2165 .

\section{Compound 3e}

Yield: $27.3 \mathrm{mg}$ (46\%); white crystalline solid; $\mathrm{mp} 156.8-158.6^{\circ} \mathrm{C}$. IR (film): 3070, 3029, 2980, 2936, 2902, 1726, 1674, 1644, 1601, $1484,1453,1401,1351,1315,1291,1263,1227,1186,1176,1168$ 1152, 1110, 1092, 1046, 1020, 1006, 875, 793, 773, 753, 736, 697, $658,527 \mathrm{~cm}^{-1}$.

${ }^{1} \mathrm{H} \mathrm{NMR}\left(300 \mathrm{MHz}, \mathrm{CDCl}_{3}\right): \delta=7.48-7.46(\mathrm{~m}, 1 \mathrm{H}), 7.36-7.14(\mathrm{~m}$, $6 \mathrm{H}), 7.14-6.94(\mathrm{~m}, 3 \mathrm{H}), 6.79-6.77(\mathrm{~m}, 1 \mathrm{H}), 6.68-6.62(\mathrm{~m}, 2 \mathrm{H})$, $6.34(\mathrm{~d}, J=10.0 \mathrm{~Hz}, 1 \mathrm{H}), 6.07(\mathrm{~d}, J=1.1 \mathrm{~Hz}, 1 \mathrm{H}), 5.62-5.59(\mathrm{~m}$, $1 \mathrm{H}), 5.09(\mathrm{~d}, J=1.5 \mathrm{~Hz}, 1 \mathrm{H}), 4.35(\mathrm{~d}, J=4.9 \mathrm{~Hz}, 1 \mathrm{H}), 3.90-3.85$ $(\mathrm{m}, 1 \mathrm{H}), 3.77(\mathrm{~d}, J=14.3 \mathrm{~Hz}, 1 \mathrm{H}), 3.46-3.41(\mathrm{~m}, 1 \mathrm{H}), 3.03-3.01$ $(\mathrm{m}, 1 \mathrm{H}), 0.60(\mathrm{t}, J=7.2 \mathrm{~Hz}, 3 \mathrm{H})$.

${ }^{13} \mathrm{C}$ NMR $\left(75 \mathrm{MHz}, \mathrm{CDCl}_{3}\right): \delta=172.2,169.7,161.7(\mathrm{~d}$, $J=244.3 \mathrm{~Hz}), 143.8,143.5,134.6,132.7$ (d, $J=3.7 \mathrm{~Hz}), 130.6$, $129.5,129.2$ (d, $J=8.3 \mathrm{~Hz}), 127.71,127.66,127.2,126.8,124.4$ (d, $J=3.5 \mathrm{~Hz}), 122.7(\mathrm{~d}, J=14.5 \mathrm{~Hz}), 121.2,120.9,119.0,115.1$ (d, $J=23.0 \mathrm{~Hz}), 111.7,100.3,64.5,62.5,61.4,31.7,12.8$.

HRMS (ESI): $m / z[\mathrm{M}+\mathrm{H}]^{+}$calcd for $\mathrm{C}_{29} \mathrm{H}_{26} \mathrm{FN}_{2} \mathrm{O}_{3}{ }^{+}: 469.1922$; found: 469.1929 .

\section{Compound 3f}

Yield: $28.8 \mathrm{mg}$ (49\%); white solid.

IR (film): 3065, 2964, 2927, 1726, 1671, 1646, 1600, 1577, 1485, $1471,1455,1446,1401,1367,1345,1310,1292,1261,1225,1187$, $1092,1027,928,876,795,757,732,699,664,655,633,527,488$, $463 \mathrm{~cm}^{-1}$. 
${ }^{1} \mathrm{H}$ NMR $\left(300 \mathrm{MHz}, \mathrm{CDCl}_{3}\right): \delta=7.64-7.50(\mathrm{~m}, 2 \mathrm{H}), 7.35-7.14(\mathrm{~m}$, $6 \mathrm{H}), 7.10-7.09(\mathrm{~m}, 1 \mathrm{H}), 7.05-6.94(\mathrm{~m}, 1 \mathrm{H}), 6.79-6.77(\mathrm{~m}, 1 \mathrm{H})$, 6.68-6.62 (m, $2 \mathrm{H}), 6.31(\mathrm{~d}, J=10.0 \mathrm{~Hz}, 1 \mathrm{H}), 6.09$ (d, $J=1.3 \mathrm{~Hz}$ $1 \mathrm{H}), 5.73-5.70(\mathrm{~m}, 1 \mathrm{H}), 5.12(\mathrm{~d}, J=1.5 \mathrm{~Hz}, 1 \mathrm{H}), 4.39-4.38(\mathrm{~m}$, $1 \mathrm{H}), 4.04(\mathrm{~d}, J=14.5 \mathrm{~Hz}, 1 \mathrm{H}), 3.93-3.70(\mathrm{~m}, 1 \mathrm{H}), 3.51-3.48(\mathrm{~m}$, $1 \mathrm{H}), 3.26(\mathrm{~d}, J=14.6 \mathrm{~Hz}, 1 \mathrm{H}), 0.60(\mathrm{t}, J=7.2 \mathrm{~Hz}, 3 \mathrm{H})$.

${ }^{13} \mathrm{C}$ NMR $\left(75 \mathrm{MHz}, \mathrm{CDCl}_{3}\right): \delta=172.2,169.5,144.1,143.8,135.5$, $134.5,132.9,132.1,130.6,129.5,129.0,127.8,127.7,127.5,127.2$ $126.8,121.3,120.9,119.7,111.7,101.0,65.1,62.1,61.4,38.8$, 12.8 .

HRMS (ESI): $m / z[\mathrm{M}+\mathrm{H}]^{+}$calcd for $\mathrm{C}_{29} \mathrm{H}_{26} \mathrm{FN}_{2} \mathrm{O}_{3}{ }^{+}: 469.1922$; found: 469.1914 .

\section{Compound 3g}

Yield: $25.3 \mathrm{mg}$ (43\%); white crystalline solid; $\mathrm{mp} 134.0-135.5^{\circ} \mathrm{C}$.

IR (film): 2963, 2925, 1727, 1669, 1601, 1578, 1510, 1485, 1455, 1446, 1403, 1368, 1346, 1261, 1222, 1188, 1160, 1103, 1050, 1017, $862,839,828,794,751 \mathrm{~cm}^{-1}$.

${ }^{1} \mathrm{H}$ NMR $\left(300 \mathrm{MHz}, \mathrm{CDCl}_{3}\right): \delta=7.47-7.38(\mathrm{~m}, 2 \mathrm{H}), 7.38-7.28(\mathrm{~m}$, $3 \mathrm{H}), 7.28-7.16(\mathrm{~m}, 2 \mathrm{H}), 7.08-6.92(\mathrm{~m}, 3 \mathrm{H}), 6.86-6.59(\mathrm{~m}, 3 \mathrm{H})$, $6.33(\mathrm{~d}, J=10.0 \mathrm{~Hz}, 1 \mathrm{H}), 6.04(\mathrm{~d}, J=1.4 \mathrm{~Hz}, 1 \mathrm{H}), 5.40-5.38(\mathrm{~m}$, $1 \mathrm{H}), 4.95(\mathrm{~d}, J=1.5 \mathrm{~Hz}, 1 \mathrm{H}), 4.41-4.40(\mathrm{~m}, 1 \mathrm{H}), 3.88-3.85(\mathrm{~m}$, $1 \mathrm{H}), 3.54-3.35(\mathrm{~m}, 2 \mathrm{H}), 3.21(\mathrm{~d}, J=14.3 \mathrm{~Hz}, 1 \mathrm{H}), 0.59(\mathrm{t}$, $J=7.2 \mathrm{~Hz}, 3 \mathrm{H}$ ).

${ }^{13} \mathrm{C}$ NMR (75 MHz, $\left.\mathrm{CDCl}_{3}\right): \delta=172.1,169.6,162.2(\mathrm{~d}$, $J=246.3 \mathrm{~Hz}), 143.7,143.6,134.4,132.3(\mathrm{~d}, J=7.9 \mathrm{~Hz}), 131.5(\mathrm{~d}$, $J=3.3 \mathrm{~Hz}), 130.8,129.6,127.8,127.6,126.8,121.2,121.0,118.6$, $115.3(\mathrm{~d}, J=21.0 \mathrm{~Hz}), 111.9,101.0,64.6,63.1,61.3,39.7,12.8$.

HRMS (ESI): $m / z[\mathrm{M}+\mathrm{H}]^{+}$calcd for $\mathrm{C}_{29} \mathrm{H}_{26} \mathrm{FN}_{2} \mathrm{O}_{3}{ }^{+}: 469.1922$; found: 469.1915

\section{Compound 3h}

Yield: $22.1 \mathrm{mg}$ (36\%); white crystalline solid; $\mathrm{mp} 185.7-187.1^{\circ} \mathrm{C}$. IR (film): 3071, 3030, 2964, 2928, 2899, 1725, 1670, 1643, 1601, $1483,1473,1447,1401,1373,1351,1311,1291,1263,1225,1186$, $1155,1110,1094,1081,1046,1018,877,827,793,775,757,733$, $695,657,631,463 \mathrm{~cm}^{-1}$.

${ }^{1} \mathrm{H}$ NMR $\left(300 \mathrm{MHz}, \mathrm{CDCl}_{3}\right): \delta=7.63-7.61(\mathrm{~m}, 1 \mathrm{H}), 7.48-7.38(\mathrm{~m}$, $1 \mathrm{H}), 7.35-7.24(\mathrm{~m}, 1 \mathrm{H}), 7.24-7.14(\mathrm{~m}, 6 \mathrm{H}), 7.07-6.94(\mathrm{~m}, 1 \mathrm{H})$, 6.86-6.56 (m, $3 \mathrm{H}), 6.32(\mathrm{~d}, J=10.0 \mathrm{~Hz}, 1 \mathrm{H}), 6.09(\mathrm{~d}, J=1.2 \mathrm{~Hz}$, $1 \mathrm{H}), 5.70-5.68(\mathrm{~m}, 1 \mathrm{H}), 5.13(\mathrm{~d}, J=1.5 \mathrm{~Hz}, 1 \mathrm{H}), 4.34-4.32(\mathrm{~m}$, $1 \mathrm{H}), 4.03(\mathrm{~d}, J=14.5 \mathrm{~Hz}, 1 \mathrm{H}), 3.92-3.89(\mathrm{~m}, 1 \mathrm{H}), 3.50-3.47(\mathrm{~m}$, $1 \mathrm{H}), 3.18(\mathrm{~d}, J=14.5 \mathrm{~Hz}, 1 \mathrm{H}), 0.60(\mathrm{t}, J=7.2 \mathrm{~Hz}, 3 \mathrm{H})$.

${ }^{13} \mathrm{C} \mathrm{NMR}\left(75 \mathrm{MHz}, \mathrm{CDCl}_{3}\right): \delta=172.2,169.6,143.9,143.8,135.8$, 134.5, 133.6, 132.2, 130.5, 129.5, 129.4, 128.8, 127.7, 127.5, 127.2, $127.1,126.7,121.2,120.9,119.4,111.7,100.9,65.0,62.0,61.4$, $35.9,12.8$.

HRMS (ESI): $m / z[\mathrm{M}+\mathrm{H}]^{+}$calcd for $\mathrm{C}_{29} \mathrm{H}_{26} \mathrm{ClN}_{2} \mathrm{O}_{3}{ }^{+}: 485.1626$; found: 485.1621 .

\section{Compound 3i}

Yield: $16.2 \mathrm{mg}$ (27\%); white solid.

IR (film): 3058, 3024, 2978, 2964, 2933, 2902, 1726, 1669, 1599, $1575,1485,1455,1446,1402,1368,1344,1312,1295,1262,1225$, $1188,1095,1049,1017,883,863,786,751,725,695,671,662$, $650,638,629,617,605 \mathrm{~cm}^{-1}$.

${ }^{1} \mathrm{H}$ NMR $\left(300 \mathrm{MHz}, \mathrm{CDCl}_{3}\right): \delta=7.48-7.40(\mathrm{~m}, 2 \mathrm{H}), 7.40-7.17(\mathrm{~m}$, $7 \mathrm{H}), 7.08-6.95(\mathrm{~m}, 1 \mathrm{H}), 6.81-6.79(\mathrm{~m}, 1 \mathrm{H}), 6.70-6.66(\mathrm{~m}, 2 \mathrm{H})$, $6.35(\mathrm{~d}, J=10.0 \mathrm{~Hz}, 1 \mathrm{H}), 6.03(\mathrm{~d}, J=1.5 \mathrm{~Hz}, 1 \mathrm{H}), 5.41-5.38(\mathrm{~m}$, $1 \mathrm{H}), 4.96(\mathrm{~d}, J=1.6 \mathrm{~Hz}, 1 \mathrm{H}), 4.42-4.40(\mathrm{~m}, 1 \mathrm{H}), 3.89-3.86(\mathrm{~m}$, $1 \mathrm{H}), 3.57-3.34(\mathrm{~m}, 2 \mathrm{H}), 3.21(\mathrm{~d}, J=14.2 \mathrm{~Hz}, 1 \mathrm{H}), 0.61(\mathrm{t}$, $J=7.2 \mathrm{~Hz}, 3 \mathrm{H})$.

${ }^{13} \mathrm{C} \mathrm{NMR}\left(75 \mathrm{MHz}, \mathrm{CDCl}_{3}\right): \delta=172.0,169.6,143.7,143.5,137.9$, $134.4,134.3,130.85,130.79,129.7,129.6,128.9,127.9,127.8$,
$127.6,126.8,121.2,121.0,118.5,111.9,101.1,64.4,63.2,61.4$, $40.2,12.8$

HRMS (ESI): $m / z[\mathrm{M}+\mathrm{H}]^{+}$calcd for $\mathrm{C}_{29} \mathrm{H}_{26} \mathrm{ClN}_{2} \mathrm{O}_{3}{ }^{+}$: 485.1626; found: 485.1624 .

\section{Compound 3j}

Yield: $23.5 \mathrm{mg}$ (39\%); white crystalline solid; mp 155.9-157.2 ${ }^{\circ} \mathrm{C}$. IR (film): 2980, 2347, 1726, 1670, 1600, 1578, 1486, 1455, 1446, 1403, 1368, 1344, 1294, 1261, 1224, 1183, 1094, 1046, 1016, 909, $866,840,753,697,650,528,464 \mathrm{~cm}^{-1}$.

${ }^{1} \mathrm{H}$ NMR $\left(300 \mathrm{MHz}, \mathrm{CDCl}_{3}\right): \delta=7.49-7.39(\mathrm{~m}, 2 \mathrm{H}), 7.39-7.18(\mathrm{~m}$, $7 \mathrm{H}), 7.09-6.96(\mathrm{~m}, 1 \mathrm{H}), 6.80-6.77(\mathrm{~m}, 1 \mathrm{H}), 6.76-6.63(\mathrm{~m}, 2 \mathrm{H})$, $6.34(\mathrm{~d}, J=10.0 \mathrm{~Hz}, 1 \mathrm{H}), 6.04(\mathrm{~d}, J=1.5 \mathrm{~Hz}, 1 \mathrm{H}), 5.40-5.38(\mathrm{~m}$, $1 \mathrm{H}), 4.95(\mathrm{~d}, J=1.5 \mathrm{~Hz}, 1 \mathrm{H}), 4.39-4.38(\mathrm{~m}, 1 \mathrm{H}), 3.88-3.85(\mathrm{~m}$, $1 \mathrm{H}), 3.52-3.42(\mathrm{~m}, 1 \mathrm{H}), 3.38(\mathrm{~d}, J=14.2 \mathrm{~Hz}, 1 \mathrm{H}), 3.21(\mathrm{~d}$, $J=14.2 \mathrm{~Hz}, 1 \mathrm{H}), 0.59$ (t, $J=7.2 \mathrm{~Hz}, 3 \mathrm{H})$.

${ }^{13} \mathrm{C} \mathrm{NMR}\left(75 \mathrm{MHz}, \mathrm{CDCl}_{3}\right): \delta=172.0,169.7,143.7,143.5,134.33$, $134.29,133.4,132.2,130.8,129.6,128.6,127.85,127.81,127.6$, $126.8,121.2,121.0,118.5,111.9,101.2,64.5,63.2,61.4,39.9$, 12.8 .

HRMS (ESI): $m / z[\mathrm{M}+\mathrm{H}]^{+}$calcd for $\mathrm{C}_{29} \mathrm{H}_{26} \mathrm{ClN}_{2} \mathrm{O}_{3}{ }^{+}: 485.1626$; found: 485.1605 .

\section{Compound 3k}

Yield: $21.72 \mathrm{mg}$ (33\%); white crystalline solid; $\mathrm{mp} 192.4-193.9^{\circ} \mathrm{C}$. IR (film): 3064, 2984, 2936, 2902, 1727, 1673, 1645, 1600, 1578, $1485,1471,1455,1446,1401,1368,1345,1310,1292,1261,1224$, $1187,1110,1090,1044,1027,1010,929,909,866,827,756,732$, $698,655,634 \mathrm{~cm}^{-1}$.

${ }^{1} \mathrm{H}$ NMR $\left(300 \mathrm{MHz}, \mathrm{CDCl}_{3}\right): \delta=7.64-7.50(\mathrm{~m}, 2 \mathrm{H}), 7.34-7.15(\mathrm{~m}$, $6 \mathrm{H}), 7.10-7.05(\mathrm{~m}, 1 \mathrm{H}), 7.01-7.00(\mathrm{~m}, 1 \mathrm{H}), 6.79-6.70(\mathrm{~m}, 1 \mathrm{H})$, $6.68-6.62(\mathrm{~m}, 2 \mathrm{H}), 6.31(\mathrm{~d}, J=10.0 \mathrm{~Hz}, 1 \mathrm{H}), 6.09(\mathrm{~d}, J=1.3 \mathrm{~Hz}$, $1 \mathrm{H}), 5.73-5.70(\mathrm{~m}, 1 \mathrm{H}), 5.12(\mathrm{~d}, J=1.5 \mathrm{~Hz}, 1 \mathrm{H}), 4.39-4.37(\mathrm{~m}$, $1 \mathrm{H}), 4.04(\mathrm{~d}, J=14.5 \mathrm{~Hz}, 1 \mathrm{H}), 3.93-3.89(\mathrm{~m}, 1 \mathrm{H}), 3.51-3.48(\mathrm{~m}$, $1 \mathrm{H}), 3.26(\mathrm{~d}, J=14.6 \mathrm{~Hz}, 1 \mathrm{H}), 0.60(\mathrm{t}, J=7.2 \mathrm{~Hz}, 3 \mathrm{H})$.

${ }^{13} \mathrm{C} \mathrm{NMR}\left(75 \mathrm{MHz}, \mathrm{CDCl}_{3}\right): \delta=172.2,169.5,144.1,143.8,135.5$, 134.5, 132.8, 132.1, 130.6, 129.5, 129.0, 127.8, 127.7, 127.5, 127.2, $126.8,121.3,120.9,119.6,111.7,100.9,65.1,62.0,61.4,38.8$, 12.8 .

HRMS (ESI): $m / z[\mathrm{M}+\mathrm{H}]^{+}$calcd for $\mathrm{C}_{29} \mathrm{H}_{26} \mathrm{BrN}_{2} \mathrm{O}_{3}{ }^{+}: 529.1121$; found: 529.1125 .

\section{Compound 31}

Yield: $24.8 \mathrm{mg}$ (37\%); white solid.

IR (film): 2979, 1726, 1669, 1600, 1567, 1485, 1455, 1446, 1426, $1402,1368,1343,1294,1261,1224,1188,1074,1045,1011,863$, $753,698,671 \mathrm{~cm}^{-1}$.

${ }^{1} \mathrm{H}$ NMR $\left(300 \mathrm{MHz}, \mathrm{CDCl}_{3}\right): \delta=7.52(\mathrm{t}, J=1.7 \mathrm{~Hz}, 1 \mathrm{H}), 7.48$ 7.37 (m, 3 H), 7.37-7.29 (m, $2 \mathrm{H}), 7.29-7.20(\mathrm{~m}, 2 \mathrm{H}), 7.16$ (t, $J=7.8 \mathrm{~Hz}, 1 \mathrm{H}), 7.07-6.97(\mathrm{~m}, 1 \mathrm{H}), 6.80-6.75(\mathrm{~m}, 1 \mathrm{H}), 6.77-$ $6.61(\mathrm{~m}, 2 \mathrm{H}), 6.34(\mathrm{~d}, J=10.0 \mathrm{~Hz}, 1 \mathrm{H}), 6.03(\mathrm{~d}, J=1.5 \mathrm{~Hz}, 1 \mathrm{H})$, 5.41-5.39 (m, $1 \mathrm{H}), 4.96(\mathrm{~d}, J=1.6 \mathrm{~Hz}, 1 \mathrm{H}), 4.41-4.40(\mathrm{~m}, 1 \mathrm{H})$, $3.88-3.86(\mathrm{~m}, 1 \mathrm{H}), 3.54-3.42(\mathrm{~m}, 1 \mathrm{H}), 3.43-3.33(\mathrm{~m}, 1 \mathrm{H}), 3.20$ $(\mathrm{d}, J=14.2 \mathrm{~Hz}, 1 \mathrm{H}), 0.60(\mathrm{t}, J=7.2 \mathrm{~Hz}, 3 \mathrm{H})$.

${ }^{13} \mathrm{C} \mathrm{NMR}\left(75 \mathrm{MHz}, \mathrm{CDCl}_{3}\right): \delta=172.0,169.6,143.7,143.5,138.2$, $134.4,133.7,130.8,130.5,130.0,129.6,129.4,127.88,127.82$, $127.6,126.8,122.4,121.2,121.0,118.5,111.9,101.2,64.5,63.2$, $61.4,40.1,12.8$.

HRMS (ESI): $m / z[\mathrm{M}+\mathrm{H}]^{+}$calcd for $\mathrm{C}_{29} \mathrm{H}_{26} \mathrm{BrN}_{2} \mathrm{O}_{3}{ }^{+}: 529.1121$; found: 529.1121 .

\section{Compounds $3 \mathrm{~m}$ and $4 \mathrm{~m}$}

Yield: $47.9 \mathrm{mg}(86 \%)$; white solid. 
IR (film): 3058, 2982, 2936, 1732, 1669, 1600, 1578, 1485, 1455, 1403, 1371, 1347, 1293, 1265, 1191, 1098, 1045, 1025, 981, 931, $871,755,698,665,529,463 \mathrm{~cm}^{-1}$.

${ }^{1} \mathrm{H}$ NMR $\left(300 \mathrm{MHz}, \mathrm{CDCl}_{3}\right): \delta=7.84-7.68(\mathrm{~m}, 4 \mathrm{H}), 7.44-7.27(\mathrm{~m}$, $6 \mathrm{H}), 7.13-6.97(\mathrm{~m}, 2 \mathrm{H}), 6.94-6.64(\mathrm{~m}, 6 \mathrm{H}), 6.39(\mathrm{t}, J=9.6 \mathrm{~Hz}$, $2 \mathrm{H}), 6.03-5.75(\mathrm{~m}, 4 \mathrm{H}), 5.33(\mathrm{~d}, J=1.5 \mathrm{~Hz}, 1 \mathrm{H}), 5.17(\mathrm{~d}$, $J=5.5 \mathrm{~Hz}, 1 \mathrm{H}), 5.08-4.91(\mathrm{~m}, 2 \mathrm{H}), 4.37-3.98(\mathrm{~m}, 6 \mathrm{H}), 3.98$ $3.76(\mathrm{~m}, 1 \mathrm{H}), 3.46-3.43(\mathrm{~m}, 1 \mathrm{H}), 3.09-2.79(\mathrm{~m}, 3 \mathrm{H}), 2.51(\mathrm{~d}$, $J=17.9 \mathrm{~Hz}, 1 \mathrm{H}), 1.37-1.14(\mathrm{~m}, 9 \mathrm{H}), 0.62(\mathrm{t}, J=7.2 \mathrm{~Hz}, 3 \mathrm{H})$.

${ }^{13} \mathrm{C}$ NMR $\left(75 \mathrm{MHz}, \mathrm{CDCl}_{3}\right): \delta=171.2,170.7,170.1,169.8,169.7$, $169.2,143.7,143.5,143.33,143.27,134.4,133.9,130.9,130.8$ 129.6, 129.4, 128.0, 127.8, 127.7, 127.6, 127.1, 126.9, 126.7, 122.1, $121.7,121.4,121.2,120.9,119.0,112.3,111.8,101.2,99.9,64.5$, $62.1,61.8,61.7,61.3,60.8,60.5,58.9,40.1,39.9,14.0,14.0,13.8$, 12.7.

HRMS (ESI): $m / z[\mathrm{M}+\mathrm{H}]^{+}$calcd for $\mathrm{C}_{26} \mathrm{H}_{27} \mathrm{~N}_{2} \mathrm{O}_{5}^{+}: 447.1914$; found: 447.1915 .

\section{Compound 4a}

Yield: $23.4 \mathrm{mg}$ (42\%); white crystalline solid; $\mathrm{mp} 152.3-153.6^{\circ} \mathrm{C}$. IR (film): 2957, 2928, 2855, 2347, 1728, 1668, 1600, 1578, 1527, 1484, 1454, 1386, 1339, 1277, 1215, 1184, 1117, 1074, 820, 772, $751,699,660 \mathrm{~cm}^{-1}$.

${ }^{1} \mathrm{H}$ NMR $\left(300 \mathrm{MHz}, \mathrm{CDCl}_{3}\right): \delta=7.79-7.67(\mathrm{~m}, 2 \mathrm{H}), 7.33-7.25(\mathrm{~m}$, $3 \mathrm{H}), 7.21-7.04(\mathrm{~m}, 4 \mathrm{H}), 7.00-6.71(\mathrm{~m}, 5 \mathrm{H}), 6.54(\mathrm{~d}, J=10.0 \mathrm{~Hz}$, $1 \mathrm{H}), 6.04-5.94(\mathrm{~m}, 1 \mathrm{H}), 5.90-5.87(\mathrm{~m}, 1 \mathrm{H}), 4.78(\mathrm{~d}, J=5.2 \mathrm{~Hz}$, $1 \mathrm{H}), 4.35(\mathrm{~s}, 1 \mathrm{H}), 4.22-4.18(\mathrm{~m}, 2 \mathrm{H}), 3.43(\mathrm{~d}, J=13.9 \mathrm{~Hz}, 1 \mathrm{H})$, $2.72(\mathrm{~d}, J=13.9 \mathrm{~Hz}, 1 \mathrm{H}), 1.27(\mathrm{t}, J=7.1 \mathrm{~Hz}, 3 \mathrm{H})$.

${ }^{13} \mathrm{C} \mathrm{NMR}\left(75 \mathrm{MHz}, \mathrm{CDCl}_{3}\right): \delta=170.9,169.4,143.6,140.6,136.0$, $134.6,130.74,130.71,129.6,128.7,128.0,127.7,127.4,127.2$, $126.6,121.9,121.8,120.2,111.8,103.9,65.0,62.1,61.6,38.5$, 13.9.

HRMS (ESI): $m / z[\mathrm{M}+\mathrm{H}]^{+}$calcd for $\mathrm{C}_{29} \mathrm{H}_{27} \mathrm{~N}_{2} \mathrm{O}_{3}{ }^{+}: 451.2016$; found: 451.2008 .

\section{Compound $4 \mathrm{~b}$}

Yield: $32.85 \mathrm{mg}$ (57\%); white crystalline solid; $\mathrm{mp} 147.3-148.2{ }^{\circ} \mathrm{C}$. IR (film): 3058, 2964, 1739, 1665, 1600, 1578, 1484, 1455, 1401, $1349,1278,1221,1181,1113,1058,1044,912,879,796,754,696$, $662 \mathrm{~cm}^{-1}$.

${ }^{1} \mathrm{H}$ NMR $\left(300 \mathrm{MHz}, \mathrm{CDCl}_{3}\right): \delta=7.79-7.68(\mathrm{~m}, 2 \mathrm{H}), 7.42-7.26(\mathrm{~m}$, $3 \mathrm{H}), 7.15-6.86(\mathrm{~m}, 6 \mathrm{H}), 6.86-6.73(\mathrm{~m}, 2 \mathrm{H}), 6.58(\mathrm{~d}, J=10.0 \mathrm{~Hz}$, $1 \mathrm{H}), 6.04-5.92(\mathrm{~m}, 1 \mathrm{H}), 5.85(\mathrm{~s}, 1 \mathrm{H}), 4.72-4.70(\mathrm{~m}, 1 \mathrm{H}), 4.44$ $4.13(\mathrm{~m}, 3 \mathrm{H}), 3.37(\mathrm{~d}, J=14.3 \mathrm{~Hz}, 1 \mathrm{H}), 2.94(\mathrm{~d}, J=14.3 \mathrm{~Hz}, 1 \mathrm{H})$, $1.69(\mathrm{~s}, 3 \mathrm{H}), 1.29(\mathrm{t}, J=7.1 \mathrm{~Hz}, 3 \mathrm{H})$.

${ }^{13} \mathrm{C} \mathrm{NMR}\left(75 \mathrm{MHz}, \mathrm{CDCl}_{3}\right): \delta=171.4,169.5,143.6,140.2,138.0$, 134.7, 134.6, 130.8, 130.1, 129.8, 129.5, 128.7, 128.0, 127.8, 127.2, $126.7,125.4,121.94,121.87,120.4,112.4,103.8,65.3,62.0,61.7$, $34.5,20.0,14.0$.

HRMS (ESI): $m / z[\mathrm{M}+\mathrm{H}]^{+}$calcd for $\mathrm{C}_{30} \mathrm{H}_{29} \mathrm{~N}_{2} \mathrm{O}_{3}{ }^{+}: 465.2173$; found: 465.2166 .

\section{Compound 4c}

Yield: $22.9 \mathrm{mg}$ (39\%); white crystalline solid; $\mathrm{mp} 141.1-142.3{ }^{\circ} \mathrm{C}$. IR (film): 3026, 2979, 2924, 1717, 1664, 1599, 1577, 1483, 1454, 1446, 1401, 1367, 1349, 1276, 1230, 1208, 1187, 1164, 1096, 1051, $1018,796,783,771,751,696 \mathrm{~cm}^{-1}$.

${ }^{1} \mathrm{H}$ NMR $\left(300 \mathrm{MHz}, \mathrm{CDCl}_{3}\right): \delta=7.73(\mathrm{~d}, J=6.9 \mathrm{~Hz}, 2 \mathrm{H}), 7.32$ $7.25(\mathrm{~m}, 3 \mathrm{H}), 7.18-6.85(\mathrm{~m}, 4 \mathrm{H}), 6.80(\mathrm{t}, J=7.4 \mathrm{~Hz}, 2 \mathrm{H}), 6.68(\mathrm{~s}$, $2 \mathrm{H}), 6.53$ (d, $J=10.0 \mathrm{~Hz}, 1 \mathrm{H}), 5.99(\mathrm{~s}, 1 \mathrm{H}), 5.90-5.87(\mathrm{~m}, 1 \mathrm{H})$, $4.77(\mathrm{~d}, J=5.2 \mathrm{~Hz}, 1 \mathrm{H}), 4.41(\mathrm{~s}, 1 \mathrm{H}), 4.35-4.09(\mathrm{~m}, 2 \mathrm{H}), 3.39(\mathrm{~d}$, $J=13.9 \mathrm{~Hz}, 1 \mathrm{H}), 2.69(\mathrm{~d}, J=13.9 \mathrm{~Hz}, 1 \mathrm{H}), 2.24(\mathrm{~s}, 3 \mathrm{H}), 1.27(\mathrm{t}$, $J=7.1 \mathrm{~Hz}, 3 \mathrm{H})$.
${ }^{13} \mathrm{C} \mathrm{NMR}\left(75 \mathrm{MHz}, \mathrm{CDCl}_{3}\right): \delta=171.1,169.5,143.7,140.6,136.9$, 135.9, 134.6, 131.5, 130.8, 129.6, 128.7, 128.0, 127.8, 127.4, 127.2, 122.0, 121.8, 120.4, 111.9, 104.0, 65.0, 62.2, 61.6, 38.7, 21.3, 14.0.

HRMS (ESI): $m / z[\mathrm{M}+\mathrm{H}]^{+}$calcd for $\mathrm{C}_{30} \mathrm{H}_{29} \mathrm{~N}_{2} \mathrm{O}_{3}{ }^{+}: 465.2173$; found: 465.2168 .

\section{Compound 4d}

Yield: $20.1 \mathrm{mg}$ (34\%); white solid.

IR (film): 3054, 3023, 2981, 2924, 2414, 2379, 2358, 2344, 2324, $1727,1667,1600,1578,1515,1484,1455,1447,1402,1368,1349$, 1276, 1230, 1209, 1186, 1165, 1113, 1096, 1072, 1050, 1017, 984, $972,951,939,928,907,883,861,851,838,826,817,795,771$, $760,727,715,695,683 \mathrm{~cm}^{-1}$.

${ }^{1} \mathrm{H}$ NMR $\left(300 \mathrm{MHz}, \mathrm{CDCl}_{3}\right): \delta=7.83-7.62(\mathrm{~m}, 2 \mathrm{H}), 7.44-7.22(\mathrm{~m}$, $3 \mathrm{H}), 7.09-7.04(\mathrm{~m}, 1 \mathrm{H}), 7.03-6.86(\mathrm{~m}, 3 \mathrm{H}), 6.85-6.70(\mathrm{~m}, 4 \mathrm{H})$, $6.53(\mathrm{~d}, J=10.1 \mathrm{~Hz}, 1 \mathrm{H}), 5.98(\mathrm{~s}, 1 \mathrm{H}), 5.89-5.87(\mathrm{~m}, 1 \mathrm{H}), 4.76-$ $4.75(\mathrm{~m}, 1 \mathrm{H}), 4.40(\mathrm{~d}, J=1.0 \mathrm{~Hz}, 1 \mathrm{H}), 4.32-4.10(\mathrm{~m}, 2 \mathrm{H}), 3.39(\mathrm{~d}$, $J=13.9 \mathrm{~Hz}, 1 \mathrm{H}), 2.68(\mathrm{~d}, J=13.9 \mathrm{~Hz}, 1 \mathrm{H}), 2.27(\mathrm{~s}, 3 \mathrm{H}), 1.28$ (t, $J=7.1 \mathrm{~Hz}, 3 \mathrm{H})$.

${ }^{13} \mathrm{C}$ NMR $\left(75 \mathrm{MHz}, \mathrm{CDCl}_{3}\right): \delta=171.1,169.5,143.7,140.6,136.2$, 134.6, 132.8, 130.8, 130.6, 129.6, 128.7, 128.2, 128.0, 127.8, 127.2, 122.0, 121.8, 120.4, 111.9, 104.0, 65.0, 62.2, 61.6, 38.3, 21.0, 14.0.

HRMS (ESI): $m / z[\mathrm{M}+\mathrm{H}]^{+}$calcd for $\mathrm{C}_{30} \mathrm{H}_{29} \mathrm{~N}_{2} \mathrm{O}_{3}{ }^{+}: 465.2173$; found: 465.2163 .

\section{Compound 4e}

Yield: $28 \mathrm{mg}$ (48\%); white crystalline solid; $\mathrm{mp} 143.9-145.6{ }^{\circ} \mathrm{C}$.

IR (film): 2965, 2935, 1721, 1665, 1600, 1585, 1492, 1484, 1455, $1402,1367,1349,1290,1275,1233,1211,1181,1106,1074,1049$, $1026,930,912,884,800,755,697,662,613,526,462 \mathrm{~cm}^{-1}$.

${ }^{1} \mathrm{H}$ NMR $\left(300 \mathrm{MHz}, \mathrm{CDCl}_{3}\right): \delta=7.78-7.68(\mathrm{~m}, 2 \mathrm{H}), 7.41-7.26(\mathrm{~m}$, $3 \mathrm{H}), 7.21-7.02(\mathrm{~m}, 2 \mathrm{H}), 7.00-6.84(\mathrm{~m}, 4 \mathrm{H}), 6.84-6.74(\mathrm{~m}, 2 \mathrm{H})$, $6.59(\mathrm{~d}, J=10.0 \mathrm{~Hz}, 1 \mathrm{H}), 5.97-5.93(\mathrm{~m}, 2 \mathrm{H}), 4.84-4.83(\mathrm{~m}, 1 \mathrm{H})$, $4.37(\mathrm{~d}, J=1.1 \mathrm{~Hz}, 1 \mathrm{H}), 4.28-4.15(\mathrm{~m}, 2 \mathrm{H}), 3.50-3.33(\mathrm{~m}, 1 \mathrm{H})$, $2.90-2.73(\mathrm{~m}, 1 \mathrm{H}), 1.26(\mathrm{t}, J=7.2 \mathrm{~Hz}, 3 \mathrm{H})$.

${ }^{13} \mathrm{C}$ NMR $\left(75 \mathrm{MHz}, \mathrm{CDCl}_{3}\right): \delta=170.8,169.6,161.7(\mathrm{~d}$, $J=246.2 \mathrm{~Hz}), 143.6,140.1,134.7,132.9$ (d, $J=4.2 \mathrm{~Hz}), 130.8$, $129.7,129.0,128.6(\mathrm{~d}, J=8.2 \mathrm{~Hz}), 128.0,127.8,127.2,123.2$, $123.0(\mathrm{~d}, J=3.7 \mathrm{~Hz}), 121.7(\mathrm{~d}, J=1.2 \mathrm{~Hz}), 120.1,114.8(\mathrm{~d}$, $J=22.4 \mathrm{~Hz}), 112.0,103.4,100.0,64.9,61.8,61.1,31.3,13.8$.

HRMS (ESI): $m / z[\mathrm{M}+\mathrm{H}]^{+}$calcd for $\mathrm{C}_{29} \mathrm{H}_{26} \mathrm{FN}_{2} \mathrm{O}_{3}{ }^{+}: 469.1922$; found: 469.1902 .

\section{Compound $4 \mathbf{f}$}

Yield: $21.9 \mathrm{mg}$ (37\%); white crystalline solid; mp 141.3-142.4 ${ }^{\circ} \mathrm{C}$. IR (film): 3058, 2964, 2929, 1720, 1666, 1600, 1577, 1484, 1472, $1455,1446,1401,1349,1264,1210,1050,1022,912,880,800$, $752,697,663,549,458 \mathrm{~cm}^{-1}$.

${ }^{1} \mathrm{H}$ NMR $\left(300 \mathrm{MHz}, \mathrm{CDCl}_{3}\right): \delta=7.80-7.66(\mathrm{~m}, 2 \mathrm{H}), 7.48-7.21(\mathrm{~m}$, $4 \mathrm{H}), 7.21-6.96(\mathrm{~m}, 4 \mathrm{H}), 6.91-6.89(\mathrm{~m}, 1 \mathrm{H}), 6.85-6.71(\mathrm{~m}, 2 \mathrm{H})$, $6.60(\mathrm{~d}, J=10.0 \mathrm{~Hz}, 1 \mathrm{H}), 6.04-5.89(\mathrm{~m}, 2 \mathrm{H}), 4.82-4.80(\mathrm{~m}, 1 \mathrm{H})$, $4.39(\mathrm{~d}, J=1.0 \mathrm{~Hz}, 1 \mathrm{H}), 4.33-4.12(\mathrm{~m}, 2 \mathrm{H}), 3.60(\mathrm{t}, J=14.3 \mathrm{~Hz}$, $1 \mathrm{H}), 3.10(\mathrm{~d}, J=14.2 \mathrm{~Hz}, 1 \mathrm{H}), 1.25(\mathrm{t}, J=7.1 \mathrm{~Hz}, 3 \mathrm{H})$.

${ }^{13} \mathrm{C} \mathrm{NMR}\left(75 \mathrm{MHz}, \mathrm{CDCl}_{3}\right): \delta=171.0,169.6,143.5,140.0,135.9$, 134.7, 132.6, 131.9, 130.8, 129.5, 129.0, 128.3, 128.0, 127.8, 127.3, $126.8,126.5,121.8,121.7,120.0,112.7,103.8,65.1,62.0,61.3$, 37.2, 13.9 .

HRMS (ESI): $m / z[\mathrm{M}+\mathrm{H}]^{+}$calcd for $\mathrm{C}_{29} \mathrm{H}_{26} \mathrm{FN}_{2} \mathrm{O}_{3}{ }^{+}: 469.1922$; found: 469.1926 .

\section{Compound 4g}

Yield: $22.8 \mathrm{mg}$ (39\%); white crystalline solid; $\mathrm{mp} 169.7-170.7^{\circ} \mathrm{C}$. IR (film): 2964, 2346, 1736, 1661, 1600, 1509, 1483, 1454, 1351, $1221,1192,1160,1098,1051,1017,928,881,862,838,804,759$, $695,683,672,660,640,628 \mathrm{~cm}^{-1}$. 
${ }^{1} \mathrm{H}$ NMR $\left(300 \mathrm{MHz}, \mathrm{CDCl}_{3}\right): \delta=7.83-7.62(\mathrm{~m}, 2 \mathrm{H}), 7.44-7.20(\mathrm{~m}$ $3 \mathrm{H}), 7.10-7.07(\mathrm{~m}, 1 \mathrm{H}), 6.97-6.70(\mathrm{~m}, 7 \mathrm{H}), 6.55(\mathrm{~d}, J=10.0 \mathrm{~Hz}$, $1 \mathrm{H}), 5.98(\mathrm{~d}, J=5.2 \mathrm{~Hz}, 1 \mathrm{H}), 5.88-5.86(\mathrm{~m}, 1 \mathrm{H}), 4.79-4.78(\mathrm{~m}$, $1 \mathrm{H}), 4.31(\mathrm{~d}, J=1.1 \mathrm{~Hz}, 1 \mathrm{H}), 4.22-4.19(\mathrm{~m}, 2 \mathrm{H}), 3.41(\mathrm{~d}$ $J=14.0 \mathrm{~Hz}, 1 \mathrm{H}), 2.68$ (d, $J=14.0 \mathrm{~Hz}, 1 \mathrm{H}), 1.27$ (t, $J=7.1 \mathrm{~Hz}$ $3 \mathrm{H})$.

${ }^{13} \mathrm{C}$ NMR $\left(75 \mathrm{MHz}, \mathrm{CDCl}_{3}\right): \delta=170.9,169.5,161.8(\mathrm{~d}$ $J=245.5 \mathrm{~Hz}), 143.6,140.6,134.6,132.4(\mathrm{~d}, J=8.0 \mathrm{~Hz}), 131.7(\mathrm{~d}$, $J=3.3 \mathrm{~Hz}), 130.8,129.8,128.9,128.0,127.8,127.3,121.9,121.8$ $120.0,114.3(\mathrm{~d}, J=21.2 \mathrm{~Hz}), 111.8,103.8,65.1,62.2,61.8,37.5$, 14.0 .

HRMS (ESI): $m / z[\mathrm{M}+\mathrm{H}]^{+}$calcd for $\mathrm{C}_{29} \mathrm{H}_{26} \mathrm{FN}_{2} \mathrm{O}_{3}^{+}: 469.1922$; found: 469.1916.

\section{Compound 4h}

Yield: $33.05 \mathrm{mg}$ (55\%); white solid.

IR (film): 3059, 2982, 2936, 1720, 1666, 1600, 1577, 1484, 1455, $1446,1402,1367,1349,1272,1231,1210,1189,1167,1112,1058$, $1025,972,930,912,882,786,753,697,661,621 \mathrm{~cm}^{-1}$.

${ }^{1} \mathrm{H}$ NMR $\left(300 \mathrm{MHz}, \mathrm{CDCl}_{3}\right): \delta=7.79-7.66(\mathrm{~m}, 2 \mathrm{H}), 7.44-7.17(\mathrm{~m}$, $4 \mathrm{H}), 7.18-6.97(\mathrm{~m}, 4 \mathrm{H}), 6.98-6.85(\mathrm{~m}, 1 \mathrm{H}), 6.79(\mathrm{t}, J=7.4 \mathrm{~Hz}$, $2 \mathrm{H}), 6.61(\mathrm{~d}, J=10.0 \mathrm{~Hz}, 1 \mathrm{H}), 6.06-5.87(\mathrm{~m}, 2 \mathrm{H}), 4.82-4.80(\mathrm{~m}$, $1 \mathrm{H}), 4.37(\mathrm{~d}, J=1.0 \mathrm{~Hz}, 1 \mathrm{H}), 4.30-4.13(\mathrm{~m}, 2 \mathrm{H}), 3.54(\mathrm{~d}$ $J=14.1 \mathrm{~Hz}, 1 \mathrm{H}), 3.05(\mathrm{~d}, J=14.1 \mathrm{~Hz}, 1 \mathrm{H}), 1.25(\mathrm{t}, J=7.1 \mathrm{~Hz}$, $3 \mathrm{H})$.

${ }^{13} \mathrm{C}$ NMR $\left(75 \mathrm{MHz}, \mathrm{CDCl}_{3}\right): \delta=170.9,169.6,143.5,139.9,135.8$, 134.6, 134.0, 132.1, 130.7, 129.5, 129.1, 129.0, 128.1, 127.9, 127.7, $127.2,125.8,121.7,121.6,120.0,112.4,103.6,65.1,61.9,61.2$, 34.6, 13.8

HRMS (ESI): $m / z[\mathrm{M}+\mathrm{H}]^{+}$calcd for $\mathrm{C}_{29} \mathrm{H}_{26} \mathrm{ClN}_{2} \mathrm{O}_{3}{ }^{+}: 485.1626$; found: 485.1630 .

\section{Compound 4i}

Yield: $25 \mathrm{mg}$ (41\%); white solid.

IR (film): 3059, 3023, 2981, 2935, 2903, 1725, 1666, 1599, 1575, $1483,1455,1446,1433,1402,1368,1349,1310,1268,1231,1211$, $1187,1166,1096,1081,1049,1018,882,793,752,728,695,671$, $661,650,639,626,616,606 \mathrm{~cm}^{-1}$.

${ }^{1} \mathrm{H} \mathrm{NMR}\left(300 \mathrm{MHz}, \mathrm{CDCl}_{3}\right): \delta=7.78-7.67(\mathrm{~m}, 2 \mathrm{H}), 7.42-7.22(\mathrm{~m}$, $3 \mathrm{H}), 7.11-7.08(\mathrm{~m}, 3 \mathrm{H}), 6.95-6.87(\mathrm{~m}, 2 \mathrm{H}), 6.86-6.71(\mathrm{~m}, 3 \mathrm{H})$, $6.54(\mathrm{~d}, J=10.0 \mathrm{~Hz}, 1 \mathrm{H}), 6.04-5.96(\mathrm{~m}, 1 \mathrm{H}), 5.87-5.84(\mathrm{~m}, 1 \mathrm{H})$, 4.81-4.80 (m, $1 \mathrm{H}), 4.38(\mathrm{~d}, J=1.3 \mathrm{~Hz}, 1 \mathrm{H}), 4.33-4.11(\mathrm{~m}, 2 \mathrm{H})$, $3.38(\mathrm{~d}, J=14.0 \mathrm{~Hz}, 1 \mathrm{H}), 2.70(\mathrm{~d}, J=14.0 \mathrm{~Hz}, 1 \mathrm{H}), 1.29(\mathrm{t}$, $J=7.1 \mathrm{~Hz}, 3 \mathrm{H})$.

${ }^{13} \mathrm{C} \mathrm{NMR}\left(75 \mathrm{MHz}, \mathrm{CDCl}_{3}\right): \delta=170.8,169.5,143.6,140.7,138.2$, $134.5,133.3,130.9,129.8,129.1,129.0,128.7,128.0,127.8,127.3$, $126.9,121.9,121.8,121.0,111.9,103.7,65.0,62.1,61.9,38.1$, 14.0.

HRMS (ESI): $m / z[\mathrm{M}+\mathrm{H}]^{+}$calcd for $\mathrm{C}_{29} \mathrm{H}_{26} \mathrm{ClN}_{2} \mathrm{O}_{3}^{+}: 485.1626$; found: 485.1634 .

\section{Compound $\mathbf{4 j}$}

Yield: $15.8 \mathrm{mg}$ (26\%); white crystalline solid; $\mathrm{mp} 177.8-179.0{ }^{\circ} \mathrm{C}$. IR (film): 2982, 2935, 2361, 2342, 1725, 1664, 1599, 1577, 1483, $1454,1402,1349,1291,1268,1231,1211,1187,1107,1092,1049$, $1016,928,911,881,827,785,755,697,662,521,462 \mathrm{~cm}^{-1}$.

${ }^{1} \mathrm{H} \mathrm{NMR}\left(300 \mathrm{MHz}, \mathrm{CDCl}_{3}\right): \delta=7.86-7.54(\mathrm{~m}, 2 \mathrm{H}), 7.49-7.19(\mathrm{~m}$, $3 \mathrm{H}), 7.19-6.98(\mathrm{~m}, 3 \mathrm{H}), 6.90-6.87(\mathrm{~m}, 1 \mathrm{H}), 6.86-6.66(\mathrm{~m}, 4 \mathrm{H})$, $6.54(\mathrm{~d}, J=10.0 \mathrm{~Hz}, 1 \mathrm{H}), 6.07-5.93(\mathrm{~m}, 1 \mathrm{H}), 5.87-5.84(\mathrm{~m}, 1 \mathrm{H})$, $4.81-4.80(\mathrm{~m}, 1 \mathrm{H}), 4.32(\mathrm{~d}, J=4.2 \mathrm{~Hz}, 1 \mathrm{H}), 4.29-4.11(\mathrm{~m}, 2 \mathrm{H})$ $3.40(\mathrm{~d}, J=14.0 \mathrm{~Hz}, 1 \mathrm{H}), 2.68(\mathrm{~d}, J=14.0 \mathrm{~Hz}, 1 \mathrm{H}), 1.28(\mathrm{t}$, $J=7.1 \mathrm{~Hz}, 3 \mathrm{H})$.

${ }^{13} \mathrm{C} \mathrm{NMR}\left(75 \mathrm{MHz}, \mathrm{CDCl}_{3}\right): \delta=170.8,169.5,143.6,140.6,134.6$, $134.5,132.6,132.2,130.9,129.8,129.0,128.0,127.8,127.6,127.3$, $121.9,120.0,111.8,103.8,65.1,62.2,61.8,37.7,14.0$.
HRMS (ESI): $m / z[\mathrm{M}+\mathrm{H}]^{+}$calcd for $\mathrm{C}_{29} \mathrm{H}_{26} \mathrm{ClN}_{2} \mathrm{O}_{3}{ }^{+}:$485.1626; found: 485.1622 .

\section{Compound 4k}

Yield: $26.55 \mathrm{mg}$ (40\%); white crystalline solid; $\mathrm{mp} 136.3-138.2^{\circ} \mathrm{C}$. IR (film): 2982, 1719, 1665, 1600, 1577, 1484, 1472, 1455, 1446, $1401,1349,1270,1230,1210,1120,1049,1022,912,881,784$, $752,697,662 \mathrm{~cm}^{-1}$.

${ }^{1} \mathrm{H}$ NMR $\left(300 \mathrm{MHz}, \mathrm{CDCl}_{3}\right): \delta=7.80-7.63(\mathrm{~m}, 2 \mathrm{H}), 7.44-7.21(\mathrm{~m}$, $3 \mathrm{H}), 7.20-7.03(\mathrm{~m}, 2 \mathrm{H}), 6.98-6.72(\mathrm{~m}, 4 \mathrm{H}), 6.71-6.45(\mathrm{~m}, 3 \mathrm{H})$, $5.98(\mathrm{~d}, J=4.0 \mathrm{~Hz}, 1 \mathrm{H}), 5.87-5.84(\mathrm{~m}, 1 \mathrm{H}), 4.81-4.79(\mathrm{~m}, 1 \mathrm{H})$, $4.36(\mathrm{~d}, J=1.3 \mathrm{~Hz}, 1 \mathrm{H}), 4.34-4.13(\mathrm{~m}, 2 \mathrm{H}), 3.42(\mathrm{~d}, J=13.9 \mathrm{~Hz}$, $1 \mathrm{H}), 2.72(\mathrm{~d}, J=14.0 \mathrm{~Hz}, 1 \mathrm{H}), 1.29$ (t, $J=7.1 \mathrm{~Hz}, 3 \mathrm{H})$.

${ }^{13} \mathrm{C}$ NMR $\left(75 \mathrm{MHz}, \mathrm{CDCl}_{3}\right): \delta=170.9,169.5,143.6,140.7,134.5$, $130.8,129.8,129.0,128.9,128.8,128.0,127.8,127.3,126.64$, $126.61,121.91,121.88,120.0,117.8,117.5,113.7,113.4,111.9$, 103.7, 65.1, 62.2, 61.8, 38.2, 14.0.

HRMS (ESI): $m / z[\mathrm{M}+\mathrm{H}]^{+}$calcd for $\mathrm{C}_{29} \mathrm{H}_{26} \mathrm{BrN}_{2} \mathrm{O}_{3}{ }^{+}: 529.1121$; found: 529.1123 .

\section{Compound 41}

Yield: $21.7 \mathrm{mg}$ (33\%); white crystalline solid; $\mathrm{mp} 153.1-154.0^{\circ} \mathrm{C}$. IR (film): 3059, 2980, 2935, 1724, 1666, 1598, 1569, 1483, 1455, 1446, 1402, 1348, 1309, 1267, 1233, 1211, 1186, 1095, 1074, 1048, $1019,972,867,788,753,697,662,612 \mathrm{~cm}^{-1}$.

${ }^{1} \mathrm{H} \mathrm{NMR}\left(300 \mathrm{MHz}, \mathrm{CDCl}_{3}\right): \delta=7.83-7.64(\mathrm{~m}, 2 \mathrm{H}), 7.43-7.23(\mathrm{~m}$, $4 \mathrm{H}), 7.17-6.96(\mathrm{~m}, 3 \mathrm{H}), 6.91-6.82(\mathrm{~m}, 1 \mathrm{H}), 6.80-6.77(\mathrm{~m}, 3 \mathrm{H})$, $6.53(\mathrm{~d}, J=10.0 \mathrm{~Hz}, 1 \mathrm{H}), 6.05-5.94(\mathrm{~m}, 1 \mathrm{H}), 5.86-5.84(\mathrm{~m}, 1 \mathrm{H})$, 4.91-4.71 (m, $1 \mathrm{H}), 4.40(\mathrm{~d}, J=1.3 \mathrm{~Hz}, 1 \mathrm{H}), 4.35-4.09(\mathrm{~m}, 2 \mathrm{H})$, $3.37(\mathrm{~d}, J=14.0 \mathrm{~Hz}, 1 \mathrm{H}), 2.70(\mathrm{~d}, J=14.0 \mathrm{~Hz}, 1 \mathrm{H}), 1.29(\mathrm{t}$, $J=7.1 \mathrm{~Hz}, 3 \mathrm{H})$.

${ }^{13} \mathrm{C}$ NMR $\left(75 \mathrm{MHz}, \mathrm{CDCl}_{3}\right): \delta=170.8,169.5,143.6,140.7,138.5$, $134.5,133.8,130.9,129.8,129.6,129.0,128.0,127.8,127.3,121.9$, 121.8, 121.5, 120.0, 111.9, 103.7, 65.0, 62.1, 61.9, 38.1, 14.0.

HRMS (ESI): $m / z[\mathrm{M}+\mathrm{H}]^{+}$calcd for $\mathrm{C}_{29} \mathrm{H}_{26} \mathrm{BrN}_{2} \mathrm{O}_{3}{ }^{+}: 529.1121$; found: 529.1099 .

\section{Compound 6a}

Yield: $24.6 \mathrm{mg}$ (55\%); white solid.

IR (film): 3057, 2983, 2924, 1732, 1668, 1600, 1578, 1484, 1447, $1402,1368,1342,1291,1261,1205,1160,1096,1017,900,881$, $862,817,793,772,759,737,716,694,683,671 \mathrm{~cm}^{-1}$.

${ }^{1} \mathrm{H} \mathrm{NMR}\left(300 \mathrm{MHz} \mathrm{CDCl}_{3}\right): \delta=7.84-7.71(\mathrm{~m}, 2 \mathrm{H}), 7.48-7.27(\mathrm{~m}$, $3 \mathrm{H}), 7.19-7.02(\mathrm{~m}, 1 \mathrm{H}), 7.00-6.70(\mathrm{~m}, 3 \mathrm{H}), 6.41$ (d, J=9.8 Hz, $1 \mathrm{H}), 6.06-5.84(\mathrm{~m}, 2 \mathrm{H}), 5.19(\mathrm{~s}, 1 \mathrm{H}), 4.53-4.50(\mathrm{~m}, 1 \mathrm{H}), 4.40$ 4.12 (m, $2 \mathrm{H}), 3.90-3.88(\mathrm{~m}, 1 \mathrm{H}), 1.32(\mathrm{t}, J=7.1 \mathrm{~Hz}, 3 \mathrm{H})$.

${ }^{13} \mathrm{C}$ NMR $\left(75 \mathrm{MHz}, \mathrm{CDCl}_{3}\right): \delta=169.2,168.8,143.1,139.1,134.3$, $130.9,129.6,128.3,127.8,127.2,126.9,122.7,122.5,121.7,114.2$, $99.8,61.6,60.6,54.1,14.1$.

HRMS (ESI): $m / z[\mathrm{M}+\mathrm{H}]^{+}$calcd for $\mathrm{C}_{22} \mathrm{H}_{21} \mathrm{~N}_{2} \mathrm{O}_{3}{ }^{+}: 361.1547$; found: 361.1533 .

\section{Compound $6 \mathrm{~b}$}

Yield: $12.1 \mathrm{mg}(26 \%)$; white solid.

IR (film): 3058, 2963, 2925, 2859, 1732, 1651, 1600, 1577, 1485, 1453, 1404, 1378, 1337, 1308, 1259, 1211, 1156, 1115, 1044, 1015, $967,891,794,760,696,663,606 \mathrm{~cm}^{-1}$.

${ }^{1} \mathrm{H} \mathrm{NMR}\left(300 \mathrm{MHz} \mathrm{CDCl}_{3}\right): \delta=7.79-7.69(\mathrm{~m}, 2 \mathrm{H}), 7.45-7.28(\mathrm{~m}$, $3 \mathrm{H}), 7.15(\mathrm{td}, J=7.8,1.7 \mathrm{~Hz}, 1 \mathrm{H}), 7.02-6.93(\mathrm{~m}, 1 \mathrm{H}), 6.89-6.86$ $(\mathrm{m}, 2 \mathrm{H}), 6.42(\mathrm{~d}, J=9.9 \mathrm{~Hz}, 1 \mathrm{H}), 6.15(\mathrm{~s}, 1 \mathrm{H}), 5.90-5.88(\mathrm{~m}$, $1 \mathrm{H}), 4.41-4.38$ (m, $1 \mathrm{H}), 4.34-4.15(\mathrm{~m}, 2 \mathrm{H}), 3.82-3.80(\mathrm{~m}, 1 \mathrm{H})$, $1.65-1.63(\mathrm{~m}, 3 \mathrm{H}), 1.31(\mathrm{t}, J=7.1 \mathrm{~Hz}, 3 \mathrm{H})$. 
${ }^{13} \mathrm{C} \mathrm{NMR}\left(75 \mathrm{MHz}, \mathrm{CDCl}_{3}\right): \delta=170.2,169.5,142.8,134.4,133.9$, $130.7,129.8,128.2,127.7,127.3,126.6,122.53,122.49,121.5$, $115.9,114.4,63.2,61.5,54.3,14.2,14.0$.

HRMS (ESI): $m / z[\mathrm{M}+\mathrm{H}]^{+}$calcd for $\mathrm{C}_{23} \mathrm{H}_{23} \mathrm{~N}_{2} \mathrm{O}_{3}{ }^{+}: 375.1703$; found: 375.1711 .

\section{Compound 7a}

Yield: $13.3 \mathrm{mg}$ (30\%); white solid.

IR (film): 3059, 3027, 2962, 2933, 2902, 1727, 1665, 1600, 1578 , $1485,1455,1447,1403,1370,1346,1292,1261,1223,1176,1158$, $1102,1071,1020,896,872,862,802,775,752,727,697,671,652$, $638,628,617,606,593 \mathrm{~cm}^{-1}$.

${ }^{1} \mathrm{H}$ NMR $\left(300 \mathrm{MHz} \mathrm{CDCl}_{3}\right): \delta=7.82-7.73(\mathrm{~m}, 2 \mathrm{H}), 7.44-7.26(\mathrm{~m}$ $3 \mathrm{H}), 7.11-6.96(\mathrm{~m}, 1 \mathrm{H}), 6.83-6.78(\mathrm{~m}, 1 \mathrm{H}), 6.74-6.70(\mathrm{~m}, 2 \mathrm{H})$, $6.43(\mathrm{~d}, J=10.0 \mathrm{~Hz}, 1 \mathrm{H}), 6.09-5.98(\mathrm{~m}, 1 \mathrm{H}), 5.80-5.78(\mathrm{~m}, 1 \mathrm{H})$ 5.09-4.94 (m, $1 \mathrm{H}), 4.70-4.68(\mathrm{~m}, 1 \mathrm{H}), 3.89-3.84(\mathrm{~m}, 2 \mathrm{H}), 3.65-$ $3.62(\mathrm{~m}, 1 \mathrm{H}), 0.76(\mathrm{t}, J=7.2 \mathrm{~Hz}, 3 \mathrm{H})$.

${ }^{13} \mathrm{C}$ NMR $\left(75 \mathrm{MHz}, \mathrm{CDCl}_{3}\right): \delta=170.0,169.6,143.4,139.7,134.4$, $131.0,129.6,128.1,127.9,127.8,126.8,121.50,121.47,119.2$, 112.6, 100.4, 61.1, 60.4, 57.2, 13.2.

HRMS (ESI): $m / z[\mathrm{M}+\mathrm{H}]^{+}$calcd for $\mathrm{C}_{22} \mathrm{H}_{21} \mathrm{~N}_{2} \mathrm{O}_{3}{ }^{+}: 361.1547$; found: 361.1530 .

\section{Compound $7 \mathrm{~b}$}

Yield: $11.9 \mathrm{mg}$ (25\%); white solid.

IR (film): 2979, 1725, 1648, 1600, 1577, 1484, 1455, 1404, 1376 , 1322, 1291, 1260, 1216, 1176, 1156, 1114, 1071, 1047, 1022, 966, $888,829,796,754,706,652 \mathrm{~cm}^{-1}$.

${ }^{1} \mathrm{H}$ NMR $\left(300 \mathrm{MHz}, \mathrm{CDCl}_{3}\right): \delta=7.73-7.70(\mathrm{~m}, 2 \mathrm{H}), 7.46-7.20(\mathrm{~m}$, $3 \mathrm{H}), 7.11-7.06(\mathrm{~m}, 1 \mathrm{H}), 6.95-6.67(\mathrm{~m}, 3 \mathrm{H}), 6.52-6.50(\mathrm{~m}, 1 \mathrm{H})$, $6.43(\mathrm{~d}, J=10.0 \mathrm{~Hz}, 1 \mathrm{H}), 5.81-5.78(\mathrm{~m}, 1 \mathrm{H}), 4.59-4.56(\mathrm{~m}, 1 \mathrm{H})$, 4.00-3.81 (m, $2 \mathrm{H}), 3.65-3.59(\mathrm{~m}, 1 \mathrm{H}), 1.71-1.68(\mathrm{~m}, 3 \mathrm{H}), 0.80(\mathrm{t}$, $J=7.1 \mathrm{~Hz}, 3 \mathrm{H})$.

${ }^{13} \mathrm{C}$ NMR $\left(75 \mathrm{MHz}, \mathrm{CDCl}_{3}\right): \delta=170.2,169.9,143.6,134.7,133.8$, 130.7, 129.7, 128.0, 127.9, 127.8, 126.8, 121.7, 121.5, 119.5, 113.9, $112.9,61.0,60.6,54.5,14.6,13.4$.

HRMS (ESI): $m / z[\mathrm{M}+\mathrm{H}]^{+}$calcd for $\mathrm{C}_{23} \mathrm{H}_{23} \mathrm{~N}_{2} \mathrm{O}_{3}{ }^{+}: 375.1703$; found: 375.1713 .

\section{Compound 9a}

Yield: $16 \mathrm{mg}$ (29\%); white crystalline solid; mp $128.3-130.1^{\circ} \mathrm{C}$.

IR (film): 3029, 2964, 1725, 1661, 1631, 1494, 1454, 1409, 1368, 1340, 1262, 1237, 1200, 1084, 1042, 941, 872, 753, 700, 675, $655 \mathrm{~cm}^{-1}$.

${ }^{1} \mathrm{H}$ NMR $\left(300 \mathrm{MHz}, \mathrm{CDCl}_{3}\right): \delta=7.71(\mathrm{~d}, J=6.4 \mathrm{~Hz}, 2 \mathrm{H}), 7.51-$ $7.30(\mathrm{~m}, 3 \mathrm{H}), 7.31-6.96(\mathrm{~m}, 8 \mathrm{H}), 6.93(\mathrm{~d}, J=7.4 \mathrm{~Hz}, 1 \mathrm{H}), 6.10(\mathrm{~d}$, $J=7.7 \mathrm{~Hz}, 1 \mathrm{H}), 5.96(\mathrm{~s}, 1 \mathrm{H}), 5.37(\mathrm{~d}, J=7.8 \mathrm{~Hz}, 1 \mathrm{H}), 5.32(\mathrm{~s}$, $1 \mathrm{H}), 4.47-4.21(\mathrm{~m}, 2 \mathrm{H}), 4.19(\mathrm{~s}, 1 \mathrm{H}), 3.16(\mathrm{~s}, 2 \mathrm{H}), 1.32(\mathrm{t}$, $J=7.1 \mathrm{~Hz}, 3 \mathrm{H})$.

${ }^{13} \mathrm{C} \mathrm{NMR}\left(75 \mathrm{MHz}, \mathrm{CDCl}_{3}\right): \delta=171.5,167.7,141.6,137.2,136.2$, $134.9,131.4,131.3,130.6,129.0,128.3,128.00,127.97,127.4$ $126.9,126.8,126.4,125.2,104.9,102.0,66.7,64.5,61.8,38.0$, 14.1 .

HRMS (ESI): $m / z[\mathrm{M}+\mathrm{H}]^{+}$calcd for $\mathrm{C}_{29} \mathrm{H}_{27} \mathrm{~N}_{2} \mathrm{O}_{3}{ }^{+}$: 451.2016; found: 451.2017 .

\section{Compound $9 \mathrm{~b}$ and $10 \mathrm{~b}$}

Yield: $29 \mathrm{mg}(50 \%)$; white crystalline solid; $\mathrm{mp} 152.1-153.7^{\circ} \mathrm{C}$.

IR (film): 3061, 3023, 2979, 1726, 1662, 1631, 1601, 1577, 1492, 1447, 1408, 1368, 1340, 1278, 1221, 1200, 1098, 1059, 1037, 940, $904,872,750,699,673,567 \mathrm{~cm}^{-1}$.

${ }^{1} \mathrm{H}$ NMR $\left(300 \mathrm{MHz}, \mathrm{CDCl}_{3}\right): \delta=7.75-7.65(\mathrm{~m}, 2 \mathrm{H}), 7.55-7.46(\mathrm{~m}$, $1 \mathrm{H}), 7.46-7.27(\mathrm{~m}, 5 \mathrm{H}), 7.27-6.83(\mathrm{~m}, 13 \mathrm{H}), 6.13(\mathrm{~d}, J=7.8 \mathrm{~Hz}$, $1 \mathrm{H}), 5.98-5.97(\mathrm{~m}, 1 \mathrm{H}), 5.92(\mathrm{~d}, J=7.8 \mathrm{~Hz}, 1 \mathrm{H}), 5.80(\mathrm{~d}$,
$J=1.0 \mathrm{~Hz}, 1 \mathrm{H}), 5.44(\mathrm{~d}, J=7.8 \mathrm{~Hz}, 1 \mathrm{H}), 5.31(\mathrm{~d}, J=7.8 \mathrm{~Hz}, 1 \mathrm{H})$, $5.22(\mathrm{~s}, 1 \mathrm{H}), 4.82(\mathrm{~d}, J=10.4 \mathrm{~Hz}, 1 \mathrm{H}), 4.61(\mathrm{~d}, J=1.3 \mathrm{~Hz}, 1 \mathrm{H})$, 4.40-4.19 (m, $2 \mathrm{H}), 4.01(\mathrm{~d}, J=1.2 \mathrm{~Hz}, 1 \mathrm{H}), 3.83-3.67(\mathrm{~m}, 1 \mathrm{H})$, $3.58(\mathrm{~d}, J=14.7 \mathrm{~Hz}, 1 \mathrm{H}), 3.34-3.33(\mathrm{~m}, 1 \mathrm{H}), 3.02(\mathrm{~d}, J=14.2 \mathrm{~Hz}$, $1 \mathrm{H}), 2.43(\mathrm{~s}, 1 \mathrm{H}), 2.14(\mathrm{~s}, 3 \mathrm{H}), 1.31(\mathrm{t}, J=9.2,5.1 \mathrm{~Hz}, 3 \mathrm{H}), 0.92$ $(\mathrm{t}, J=7.1 \mathrm{~Hz}, 1 \mathrm{H})$.

${ }^{13} \mathrm{C}$ NMR $\left(75 \mathrm{MHz}, \mathrm{CDCl}_{3}\right): \delta=171.8,171.6,167.62,167.57$, 142.2, 141.1, 138.6, 137.8, 137.4, 137.1, 135.0, 134.8, 134.4, 131.3, $130.8,130.59,130.56,130.51,130.47,130.0,129.3,129.0,128.8$, $128.2,128.00,127.95,127.9,127.6,127.1,127.0,126.8,126.5$, $126.3,126.1,125.3,125.2,124.9,104.8,104.6,101.8,101.6,67.3$, 67.2, 64.8, 64.6, 61.8, 61.4, 37.7, 33.7, 21.1, 20.8, 14.0, 13.4.

HRMS (ESI): $m / z[\mathrm{M}+\mathrm{H}]^{+}$calcd for $\mathrm{C}_{30} \mathrm{H}_{29} \mathrm{~N}_{2} \mathrm{O}_{3}{ }^{+}: 465.2173$; found: 465.2175 .

\section{Compound 9c and 10c}

Yield: $43.4 \mathrm{mg}$ (75\%); white solid.

IR (film): 2963, 2926, 1727, 1667, 1634, 1603, 1491, 1447, 1408, $1368,1340,1260,1227,1203,1097,1047,1026,939,905,862$, $755,700,667,635,561 \mathrm{~cm}^{-1}$.

${ }^{1} \mathrm{H}$ NMR $\left(300 \mathrm{MHz}, \mathrm{CDCl}_{3}\right): \delta=7.71(\mathrm{dd}, J=7.9,1.6 \mathrm{~Hz}, 1 \mathrm{H})$, 7.52-7.31 (m, 3 H), 7.31-6.97 (m, $13 \mathrm{H}), 6.93(\mathrm{~d}, J=7.3 \mathrm{~Hz}, 1 \mathrm{H})$, $6.87(\mathrm{dd}, J=5.6,2.1 \mathrm{~Hz}, 1 \mathrm{H}), 6.76(\mathrm{~s}, 1 \mathrm{H}), 6.11(\mathrm{t}, J=4.7 \mathrm{~Hz}$, $1 \mathrm{H}), 5.98(\mathrm{~s}, 1 \mathrm{H}), 5.89(\mathrm{~d}, J=7.8 \mathrm{~Hz}, 1 \mathrm{H}), 5.44-5.18(\mathrm{~m}, 2 \mathrm{H})$, $5.00(\mathrm{~d}, J=1.4 \mathrm{~Hz}, 1 \mathrm{H}), 4.86(\mathrm{~s}, 1 \mathrm{H}), 4.42-4.19(\mathrm{~m}, 1 \mathrm{H}), 3.90-$ $3.60(\mathrm{~m}, 2 \mathrm{H}), 3.51(\mathrm{~d}, J=14.4 \mathrm{~Hz}, 1 \mathrm{H}), 3.34(\mathrm{~d}, J=14.4 \mathrm{~Hz}, 1 \mathrm{H})$, 3.14 (s, $1 \mathrm{H}), 2.30$ (s, $3 \mathrm{H}), 2.23(\mathrm{~s}, 1 \mathrm{H}), 1.34$ (t, J=7.1 Hz, $2 \mathrm{H})$, 0.94 (t, $J=7.2 \mathrm{~Hz}, 3 \mathrm{H})$.

${ }^{13} \mathrm{C}$ NMR $\left(75 \mathrm{MHz}, \mathrm{CDCl}_{3}\right): \delta=171.8,171.6,167.7,143.1,141.8$, $138.2,137.2,136.9,136.7,136.0,135.5,134.9,132.0,131.5,130.7$, $130.6,130.2,128.9,128.7,128.4,128.2,128.1,128.04,127.97$, $127.8,127.7,127.4,127.2,127.1,126.9,126.8,126.7,126.3,125.1$, $124.9,105.0,104.2,102.0,101.1,66.5,65.6,64.4,63.3,61.8,61.3$, 41.4, 38.3, 21.3, 14.0, 13.5.

HRMS (ESI): $m / z[\mathrm{M}+\mathrm{H}]^{+}$calcd for $\mathrm{C}_{30} \mathrm{H}_{29} \mathrm{~N}_{2} \mathrm{O}_{3}{ }^{+}: 465.2173$; found: 465.2167 .

\section{Compound 9e}

Yield: $29.9 \mathrm{mg}$ (51\%); white crystalline solid; $\mathrm{mp} 140.3-141.6{ }^{\circ} \mathrm{C}$. IR (film): 3432, 2963, 1725, 1662, 1630, 1584, 1492, 1455, 1409, $1369,1341,1262,1235,1202,1182,1104,1047,942,904,861$, $798,758,699,674 \mathrm{~cm}^{-1}$.

${ }^{1} \mathrm{H}$ NMR $\left(300 \mathrm{MHz}, \mathrm{CDCl}_{3}\right): \delta=7.72-7.69(\mathrm{~m}, 2 \mathrm{H}), 7.47-7.32(\mathrm{~m}$, $3 \mathrm{H}), 7.33-7.06(\mathrm{~m}, 5 \mathrm{H}), 7.05-6.81(\mathrm{~m}, 3 \mathrm{H}), 6.14(\mathrm{~d}, J=7.8 \mathrm{~Hz}$, $1 \mathrm{H}), 5.88(\mathrm{~d}, J=1.3 \mathrm{~Hz}, 1 \mathrm{H}), 5.48-5.28(\mathrm{~m}, 2 \mathrm{H}), 4.42-4.15(\mathrm{~m}$, $2 \mathrm{H}), 4.04(\mathrm{~d}, J=1.3 \mathrm{~Hz}, 1 \mathrm{H}), 3.45(\mathrm{~d}, J=14.1 \mathrm{~Hz}, 1 \mathrm{H}), 2.91(\mathrm{~d}$, $J=14.1 \mathrm{~Hz}, 1 \mathrm{H}), 1.31(\mathrm{t}, J=7.1 \mathrm{~Hz}, 3 \mathrm{H})$.

${ }^{13} \mathrm{C}$ NMR $\left(75 \mathrm{MHz}, \mathrm{CDCl}_{3}\right): \delta=171.4,167.6,162.3(\mathrm{~d}$, $J=245.7 \mathrm{~Hz}), 141.5,137.4,135.0,132.7$ (d, $J=4.1 \mathrm{~Hz}), 131.5$, $130.6,129.0,128.4(\mathrm{~d}, J=8.4 \mathrm{~Hz}), 128.2,128.0,127.5,126.8(\mathrm{~d}$, $J=2.4 \mathrm{~Hz}), 125.3,123.9,123.7,123.1(\mathrm{~d}, J=3.4 \mathrm{~Hz}), 114.7(\mathrm{~d}$, $J=22.6 \mathrm{~Hz}), 104.3,100.4,66.6,64.0,61.9,30.3,14.0$.

HRMS (ESI): $m / z[\mathrm{M}+\mathrm{H}]^{+}$calcd for $\mathrm{C}_{29} \mathrm{H}_{26} \mathrm{FN}_{2} \mathrm{O}_{3}{ }^{+}: 469.1922$; found: 469.1921.

\section{Compound 9f}

Yield: $8.4 \mathrm{mg}(14 \%)$; white solid.

IR (film): 3066, 2981, 2935, 1726, 1662, 1630, 1616, 1588, 1524, 1489, 1448, 1409, 1368, 1340, 1254, 1201, 1144, 1122, 1099, 1080, $1047,941,904,877,753,700,674,629 \mathrm{~cm}^{-1}$.

${ }^{1} \mathrm{H}$ NMR $\left(300 \mathrm{MHz}, \mathrm{CDCl}_{3}\right): \delta=7.75-7.64(\mathrm{~m}, 2 \mathrm{H}), 7.47-7.31(\mathrm{~m}$, $3 \mathrm{H}), 7.21-7.20(\mathrm{~m}, 1 \mathrm{H}), 7.09-7.07(\mathrm{~m}, 3 \mathrm{H}), 6.97-6.88(\mathrm{~m}, 1 \mathrm{H})$, 6.87-6.69 (m, $3 \mathrm{H}), 6.09(\mathrm{~d}, J=7.8 \mathrm{~Hz}, 1 \mathrm{H}), 5.98(\mathrm{~d}, J=1.4 \mathrm{~Hz}$, $1 \mathrm{H}), 5.37(\mathrm{~d}, J=8.0 \mathrm{~Hz}, 2 \mathrm{H}), 4.47-4.10(\mathrm{~m}, 3 \mathrm{H}), 3.16(\mathrm{~s}, 2 \mathrm{H})$, $1.35(\mathrm{t}, J=7.1 \mathrm{~Hz}, 3 \mathrm{H})$. 
${ }^{13} \mathrm{C}$ NMR (75 $\left.\mathrm{MHz}, \mathrm{CDCl}_{3}\right): \delta=171.3,167.9,162.0(\mathrm{~d}$ $J=244.5 \mathrm{~Hz}), 141.7,138.8(\mathrm{~d}, J=7.6 \mathrm{~Hz}), 137.0,134.8,131.3$, $130.7,129.1,128.6$ (d, $J=8.2 \mathrm{~Hz}), 128.2,128.0,127.8,126.9$ (d, $J=2.7 \mathrm{~Hz}), 126.83,126.7,125.3,117.9(\mathrm{~d}, J=21.4 \mathrm{~Hz}), 113.3(\mathrm{~d}$, $J=20.9 \mathrm{~Hz}), 104.9,101.6,66.5,64.4,62.0,37.8,14.0$.

HRMS (ESI): $m / z[\mathrm{M}+\mathrm{H}]^{+}$calcd for $\mathrm{C}_{29} \mathrm{H}_{26} \mathrm{FN}_{2} \mathrm{O}_{3}{ }^{+}: 469.1922$; found: 469.1931 .

\section{Compound 9g}

Yield: $13.6 \mathrm{mg}$ (23\%); white solid.

IR (film): 3430, 2963, 1721, 1663, 1629, 1601, 1509, 1446, 1368, $1341,1262,1222,1159,1098,1045,798,757,699,670 \mathrm{~cm}^{-1}$.

${ }^{1} \mathrm{H}$ NMR $\left(300 \mathrm{MHz}, \mathrm{CDCl}_{3}\right): \delta=7.77-7.63(\mathrm{~m}, 2 \mathrm{H}), 7.50-7.30(\mathrm{~m}$, $3 \mathrm{H}), 7.25-6.89(\mathrm{~m}, 6 \mathrm{H}), 6.87-6.73(\mathrm{~m}, 2 \mathrm{H}), 6.08(\mathrm{~d}, J=7.8 \mathrm{~Hz}$, $1 \mathrm{H}), 5.98(\mathrm{~d}, J=1.8 \mathrm{~Hz}, 1 \mathrm{H}), 5.36(\mathrm{~d}, J=7.1 \mathrm{~Hz}, 2 \mathrm{H}), 4.40-4.19$ $(\mathrm{m}, 2 \mathrm{H}), 4.15(\mathrm{~d}, J=9.7 \mathrm{~Hz}, 1 \mathrm{H}), 3.13(\mathrm{~s}, 2 \mathrm{H}), 1.33$ (t, $J=7.1 \mathrm{~Hz}$, $3 \mathrm{H})$.

${ }^{13} \mathrm{C}$ NMR $\left(75 \mathrm{MHz}, \mathrm{CDCl}_{3}\right): \delta=171.4,167.7,161.7(\mathrm{~d}$, $J=244.9 \mathrm{~Hz}), 141.6,137.1,134.8,132.8(\mathrm{~d}, J=7.9 \mathrm{~Hz}), 131.8(\mathrm{~d}$, $J=3.1 \mathrm{~Hz}), 131.4,130.7,129.0,128.3,128.0,127.8,126.8(\mathrm{~d}$, $J=5.7 \mathrm{~Hz}), 125.3,114.3,114.0,104.8,101.7,66.6,64.5$, 61.9, 37.0, 14.1

HRMS (ESI): $m / z[\mathrm{M}+\mathrm{H}]^{+}$calcd for $\mathrm{C}_{29} \mathrm{H}_{26} \mathrm{FN}_{2} \mathrm{O}_{3}^{+}: 469.1922$; found: 469.1920 .

\section{Compound 9h}

Yield: $22.4 \mathrm{mg}$ (37\%); white solid.

IR (film): 3066, 2964, 1724, 1662, 1630, 1572, 1493, 1475, 1446, $1409,1369,1341,1263,1238,1201,1099,1056,1028,941,904$, $860,756,698,668,623,564 \mathrm{~cm}^{-1}$.

${ }^{1} \mathrm{H} \mathrm{NMR}\left(300 \mathrm{MHz}, \mathrm{CDCl}_{3}\right): \delta=7.71-7.69(\mathrm{~m}, 2 \mathrm{H}), 7.40-7.35(\mathrm{~m}$, $4 \mathrm{H}), 7.31-7.02(\mathrm{~m}, 6 \mathrm{H}), 6.96(\mathrm{~d}, J=7.3 \mathrm{~Hz}, 1 \mathrm{H}), 6.15(\mathrm{~d}$, $J=7.8 \mathrm{~Hz}, 1 \mathrm{H}), 5.88(\mathrm{~s}, 1 \mathrm{H}), 5.50-5.16(\mathrm{~m}, 2 \mathrm{H}), 4.47-4.15(\mathrm{~m}$, $2 \mathrm{H}), 4.02(\mathrm{~s}, 1 \mathrm{H}), 3.56(\mathrm{~d}, J=14.0 \mathrm{~Hz}, 1 \mathrm{H}), 3.08(\mathrm{~d}, J=14.0 \mathrm{~Hz}$, $1 \mathrm{H}), 1.29(\mathrm{t}, J=7.1 \mathrm{~Hz}, 3 \mathrm{H})$

${ }^{13} \mathrm{C}$ NMR $\left(75 \mathrm{MHz}, \mathrm{CDCl}_{3}\right): \delta=171.4,167.5,141.0,137.6,137.0$, $135.0,134.6,132.5,131.5,130.6,129.10,129.04,128.2,128.0$ $127.9,127.6,126.7,126.6,126.0,125.3,104.0,100.8,66.8,64.5$, $61.9,34.0,14.0$.

HRMS (ESI): $m / z[\mathrm{M}+\mathrm{H}]^{+}$calcd for $\mathrm{C}_{29} \mathrm{H}_{26} \mathrm{ClN}_{2} \mathrm{O}_{3}{ }^{+}: 485.1626$; found: 485.1614 .

\section{Compound 9i}

Yield: $14.1 \mathrm{mg}$ (23\%); white solid.

IR (film): 3064, 2980, 1724, 1662, 1630, 1599, 1573, 1477, 1447, $1368,1341,1262,1201,1081,1046,940,880,771,700,671 \mathrm{~cm}^{-1}$.

${ }^{1} \mathrm{H}$ NMR $\left(300 \mathrm{MHz}, \mathrm{CDCl}_{3}\right): \delta=7.77-7.63(\mathrm{~m}, 2 \mathrm{H}), 7.51-7.31(\mathrm{~m}$, $3 \mathrm{H}), 7.21-7.20(\mathrm{~m}, 1 \mathrm{H}), 7.16-7.00(\mathrm{~m}, 4 \mathrm{H}), 7.00-6.88(\mathrm{~m}, 3 \mathrm{H})$, $6.08(\mathrm{~d}, J=7.8 \mathrm{~Hz}, 1 \mathrm{H}), 6.00(\mathrm{~d}, J=1.4 \mathrm{~Hz}, 1 \mathrm{H}), 5.37(\mathrm{~s}, 1 \mathrm{H})$, $5.35(\mathrm{~s}, 1 \mathrm{H}), 4.51-4.12(\mathrm{~m}, 3 \mathrm{H}), 3.14(\mathrm{~s}, 2 \mathrm{H}), 1.35$ (t, $J=7.1 \mathrm{~Hz}$, $3 \mathrm{H})$.

${ }^{13} \mathrm{C} \mathrm{NMR}\left(75 \mathrm{MHz}, \mathrm{CDCl}_{3}\right): \delta=171.3,167.7,141.8,138.3,137.0$, $134.8,133.1,131.33,131.25,130.7,129.3,129.1,128.5,128.3$ $128.0,127.8,126.8,126.7,126.6,125.3,104.9,101.6,66.3,64.4$, $62.0,37.9,14.0$.

HRMS (ESI): $m / z[\mathrm{M}+\mathrm{H}]^{+}$calcd for $\mathrm{C}_{29} \mathrm{H}_{26} \mathrm{ClN}_{2} \mathrm{O}_{3}{ }^{+}: 485.1626$; found: 485.1604 .

\section{Compound 91}

Yield: $20.7 \mathrm{mg}$ (31\%); white solid.

IR (film): 3063, 2980, 1725, 1662, 1630, 1597, 1569, 1492, 1475, $1447,1368,1340,1239,1201,1096,1073,1046,941,878,769$, $699,664 \mathrm{~cm}^{-1}$.
${ }^{1} \mathrm{H} \mathrm{NMR}\left(300 \mathrm{MHz}, \mathrm{CDCl}_{3}\right): \delta=7.73-7.66(\mathrm{~m}, 2 \mathrm{H}), 7.47-7.33(\mathrm{~m}$, $3 \mathrm{H}), 7.28-7.17(\mathrm{~m}, 2 \mathrm{H}), 7.14-7.03(\mathrm{~m}, 3 \mathrm{H}), 7.03-6.89(\mathrm{~m}, 3 \mathrm{H})$, $6.08(\mathrm{~d}, J=7.8 \mathrm{~Hz}, 1 \mathrm{H}), 6.01(\mathrm{~d}, J=1.4 \mathrm{~Hz}, 1 \mathrm{H}), 5.46-5.25(\mathrm{~m}$, $2 \mathrm{H}), 4.46-4.15(\mathrm{~m}, 3 \mathrm{H}), 3.14$ (s, $2 \mathrm{H}), 1.35$ (t, $J=7.1 \mathrm{~Hz}, 3 \mathrm{H})$.

${ }^{13} \mathrm{C} \mathrm{NMR}\left(75 \mathrm{MHz}, \mathrm{CDCl}_{3}\right): \delta=171.3,167.7,141.8,138.6,137.0$, 134.8, 134.1, 131.3, 130.7, 129.7, 129.5, 129.1, 128.8, 128.2, 128.0, $127.8,126.8,126.6,125.3,121.4,104.9,101.6,66.3,64.3,62.0$, $37.9,14.0$.

HRMS (ESI): $m / z[\mathrm{M}+\mathrm{H}]^{+}$calcd for $\mathrm{C}_{29} \mathrm{H}_{26} \mathrm{BrN}_{2} \mathrm{O}_{3}{ }^{+}: 529.1121$; found: 529.1117 .

\section{Compound 9m}

Yield: $18.2 \mathrm{mg}(32 \%)$; white solid.

IR (film): 3064, 2982, 2936, 1732, 1667, 1631, 1601, 1579, 1492, $1447,1408,1370,1344,1261,1226,1182,1093,1059,1028,935$, $907,878,755,701,649 \mathrm{~cm}^{-1}$.

${ }^{1} \mathrm{H} \mathrm{NMR}\left(300 \mathrm{MHz}, \mathrm{CDCl}_{3}\right): \delta=7.69-7.50(\mathrm{~m}, 2 \mathrm{H}), 7.47-7.31(\mathrm{~m}$, $3 \mathrm{H}), 7.30-7.23(\mathrm{~m}, 1 \mathrm{H}), 7.23-7.04(\mathrm{~m}, 2 \mathrm{H}), 6.90(\mathrm{~d}, J=7.4 \mathrm{~Hz}$, $1 \mathrm{H}), 6.04(\mathrm{~d}, J=7.8 \mathrm{~Hz}, 2 \mathrm{H}), 5.50(\mathrm{~s}, 1 \mathrm{H}), 5.46(\mathrm{~d}, J=7.8 \mathrm{~Hz}$, $1 \mathrm{H}), 5.39(\mathrm{~d}, J=1.0 \mathrm{~Hz}, 1 \mathrm{H}), 4.48-4.11(\mathrm{~m}, 2 \mathrm{H}), 3.75-3.41(\mathrm{~m}$, $2 \mathrm{H}), 3.12-2.90(\mathrm{~m}, 2 \mathrm{H}), 1.35(\mathrm{t}, J=7.1 \mathrm{~Hz}, 3 \mathrm{H}), 0.98(\mathrm{t}$, $J=7.2 \mathrm{~Hz}, 3 \mathrm{H})$.

${ }^{13} \mathrm{C} \mathrm{NMR}\left(75 \mathrm{MHz}, \mathrm{CDCl}_{3}\right): \delta=170.2,169.3,167.7,143.8,137.7$, $134.5,130.9,130.8,130.2,129.0,128.3,128.0,126.4,126.2,124.8$, 106.3, 100.0, 63.4, 61.9, 60.5, 60.3, 40.4, 14.0, 13.8 .

HRMS (ESI): $m / z[\mathrm{M}+\mathrm{H}]^{+}$calcd for $\mathrm{C}_{26} \mathrm{H}_{27} \mathrm{~N}_{2} \mathrm{O}_{5}{ }^{+}: 447.1914$; found: 447.1916 .

\section{Compound 10a}

Yield: $23.8 \mathrm{mg}$ (42\%); white crystalline solid; $\mathrm{mp} 173.5-174.8^{\circ} \mathrm{C}$. IR (film): 3062, 3026, 2963, 1728, 1666, 1634, 1601, 1572, 1494, $1453,1408,1368,1340,1286,1260,1226,1200,1096,1017,939$, $906,862,769,700,664,635 \mathrm{~cm}^{-1}$.

${ }^{1} \mathrm{H} \mathrm{NMR}\left(300 \mathrm{MHz}, \mathrm{CDCl}_{3}\right): \delta=7.47-7.38(\mathrm{~m}, 2 \mathrm{H}), 7.38-7.20(\mathrm{~m}$, $8 \mathrm{H}), 7.20-7.11(\mathrm{~m}, 3 \mathrm{H}), 6.91-6.80(\mathrm{~m}, 1 \mathrm{H}), 6.12(\mathrm{~d}, J=1.4 \mathrm{~Hz}$, $1 \mathrm{H}), 5.89(\mathrm{~d}, J=7.8 \mathrm{~Hz}, 1 \mathrm{H}), 5.27(\mathrm{~d}, J=7.8 \mathrm{~Hz}, 1 \mathrm{H}), 4.97(\mathrm{~d}$, $J=1.4 \mathrm{~Hz}, 1 \mathrm{H}), 4.84(\mathrm{~s}, 1 \mathrm{H}), 3.90-3.61(\mathrm{~m}, 2 \mathrm{H}), 3.53(\mathrm{~d}$, $J=14.3 \mathrm{~Hz}, 1 \mathrm{H}), 3.36(\mathrm{~d}, J=14.4 \mathrm{~Hz}, 1 \mathrm{H}), 0.93(\mathrm{t}, J=7.1 \mathrm{~Hz}$, $3 \mathrm{H})$.

${ }^{13} \mathrm{C}$ NMR $\left(75 \mathrm{MHz}, \mathrm{CDCl}_{3}\right): \delta=171.8,167.7,142.9,136.9,135.6$, $134.8,130.9,130.7,130.3,128.8,128.5,127.8,127.6,127.5,127.4$, $126.8,126.3,125.0,104.3,101.2,65.5,63.5,61.4,41.4,13.4$.

HRMS (ESI): $m / z[\mathrm{M}+\mathrm{H}]^{+}$calcd for $\mathrm{C}_{29} \mathrm{H}_{27} \mathrm{~N}_{2} \mathrm{O}_{3}{ }^{+}: 451.2016$; found: 451.2025 .

\section{Compound 10e}

Yield: $28 \mathrm{mg}$ (47\%); white crystalline solid; $\mathrm{mp} 176.0-177.9{ }^{\circ} \mathrm{C}$.

IR (film): 3064, 2963, 1729, 1668, 1636, 1601, 1581, 1491, 1453, $1407,1368,1339,1288,1260,1227,1179,1097,1046,1016,938$, $907,871,792,759,699,663,634 \mathrm{~cm}^{-1}$.

${ }^{1} \mathrm{H}$ NMR $\left(300 \mathrm{MHz}, \mathrm{CDCl}_{3}\right): \delta=7.54-7.40(\mathrm{~m}, 1 \mathrm{H}), 7.33-7.31(\mathrm{~m}$, $2 \mathrm{H}), 7.28-7.18(\mathrm{~m}, 3 \mathrm{H}), 7.18-7.00(\mathrm{~m}, 6 \mathrm{H}), 6.86-6.85(\mathrm{~m}, 1 \mathrm{H})$, $6.10(\mathrm{~d}, J=1.3 \mathrm{~Hz}, 1 \mathrm{H}), 5.85(\mathrm{~d}, J=7.8 \mathrm{~Hz}, 1 \mathrm{H}), 5.27$ (d, $J=7.8 \mathrm{~Hz}, 1 \mathrm{H}), 5.07(\mathrm{~d}, J=1.5 \mathrm{~Hz}, 1 \mathrm{H}), 4.81(\mathrm{~s}, 1 \mathrm{H}), 3.91-3.57$ $(\mathrm{m}, 3 \mathrm{H}), 3.17(\mathrm{~d}, J=15.8 \mathrm{~Hz}, 1 \mathrm{H}), 0.92(\mathrm{t}, J=7.1 \mathrm{~Hz}, 3 \mathrm{H})$.

${ }^{13} \mathrm{C}$ NMR $\left(75 \mathrm{MHz}, \mathrm{CDCl}_{3}\right): \delta=171.7,167.3,161.6(\mathrm{~d}$, $J=243.9 \mathrm{~Hz}), 143.2,136.4,134.7,132.3(\mathrm{~d}, J=3.6 \mathrm{~Hz}), 130.6$, $130.3,129.2$ (d, $J=8.4 \mathrm{~Hz}), 128.7,127.8,127.4,127.0,126.9$ (d, $J=5.9 \mathrm{~Hz}), 124.9,124.5(\mathrm{~d}, J=3.5 \mathrm{~Hz}), 122.5,122.3,115.3(\mathrm{~d}$, $J=23.4 \mathrm{~Hz}$ ), 104.4, 100.8, 65.9, 63.38, 63.35, 61.4, 33.29, 33.26, 13.4.

HRMS (ESI): $m / z[\mathrm{M}+\mathrm{H}]^{+}$calcd for $\mathrm{C}_{29} \mathrm{H}_{26} \mathrm{FN}_{2} \mathrm{O}_{3}{ }^{+}: 469.1922$; found: 469.1931 . 


\section{Compound 10f}

Yield: $27.5 \mathrm{mg}$ (47\%); white crystalline solid; $\mathrm{mp} 155.1-156.7^{\circ} \mathrm{C}$. IR (film): 3023, 2963, 2925, 1728, 1667, 1634, 1601, 1573, 1515, $1493,1446,1408,1368,1340,1286,1260,1225,1202,1096,1045$, $1023,939,906,863,802,769,699,665,634 \mathrm{~cm}^{-1}$.

${ }^{1} \mathrm{H}$ NMR $\left(300 \mathrm{MHz}, \mathrm{CDCl}_{3}\right): \delta=7.41-7.19(\mathrm{~m}, 7 \mathrm{H}), 7.20-7.05(\mathrm{~m}$, $5 \mathrm{H}), 6.90-6.77(\mathrm{~m}, 1 \mathrm{H}), 6.11(\mathrm{~d}, J=1.3 \mathrm{~Hz}, 1 \mathrm{H}), 5.88(\mathrm{~d}$, $J=7.8 \mathrm{~Hz}, 1 \mathrm{H}), 5.26(\mathrm{~d}, J=7.8 \mathrm{~Hz}, 1 \mathrm{H}), 4.96(\mathrm{~d}, J=1.4 \mathrm{~Hz}, 1 \mathrm{H})$, 4.84 (s, $1 \mathrm{H}), 3.91-3.57$ (m, $2 \mathrm{H}), 3.49$ (d, $J=14.4 \mathrm{~Hz}, 1 \mathrm{H}), 3.31$ $(\mathrm{d}, J=14.4 \mathrm{~Hz}, 1 \mathrm{H}), 0.92(\mathrm{t}, J=7.2 \mathrm{~Hz}, 3 \mathrm{H})$.

${ }^{13} \mathrm{C}$ NMR $\left(75 \mathrm{MHz}, \mathrm{CDCl}_{3}\right): \delta=171.8,167.7,142.9,137.0,136.9$, 134.9, 132.4, 130.8, 130.7, 130.3, 129.2, 128.8, 127.8, 127.7, 127.6, 126.8, 126.3, 125.0, 104.3, 101.0, 65.5, 63.4, 61.4, 40.9, 20.9, 13.5.

HRMS (ESI): $m / z[\mathrm{M}+\mathrm{H}]^{+}$calcd for $\mathrm{C}_{29} \mathrm{H}_{26} \mathrm{FN}_{2} \mathrm{O}_{3}{ }^{+}: 469.1922$; found: 469.1917.

\section{Compound 10g}

Yield: $27.9 \mathrm{mg}$ (48\%); white crystalline solid; $\mathrm{mp} 146.0-147.0^{\circ} \mathrm{C}$. IR (film): 3064, 2961, 2929, 1727, 1665, 1635, 1602, 1573, 1510, $1493,1446,1408,1368,1340,1286,1223,1160,1098,1046,1016$, $939,906,863,837,756,699,664,633 \mathrm{~cm}^{-1}$.

${ }^{1} \mathrm{H}$ NMR $\left(300 \mathrm{MHz}, \mathrm{CDCl}_{3}\right): \delta=7.45-7.24(\mathrm{~m}, 7 \mathrm{H}), 7.24-7.09(\mathrm{~m}$, $3 \mathrm{H}), 7.07-6.94(\mathrm{~m}, 2 \mathrm{H}), 6.88(\mathrm{~d}, J=6.6 \mathrm{~Hz}, 1 \mathrm{H}), 6.11(\mathrm{~d}$, $J=1.4 \mathrm{~Hz}, 1 \mathrm{H}), 5.91(\mathrm{~d}, J=7.8 \mathrm{~Hz}, 1 \mathrm{H}), 5.30(\mathrm{~d}, J=7.8 \mathrm{~Hz}, 1 \mathrm{H})$ $4.90(\mathrm{~d}, J=1.4 \mathrm{~Hz}, 1 \mathrm{H}), 4.77(\mathrm{~s}, 1 \mathrm{H}), 3.88-3.57(\mathrm{~m}, 2 \mathrm{H}), 3.51$ (d, $J=14.4 \mathrm{~Hz}, 1 \mathrm{H}), 3.29(\mathrm{~d}, J=14.4 \mathrm{~Hz}, 1 \mathrm{H}), 0.93(\mathrm{t}, J=7.2 \mathrm{~Hz}$, $3 \mathrm{H})$.

${ }^{13} \mathrm{C}$ NMR $\left(75 \mathrm{MHz}, \mathrm{CDCl}_{3}\right): \delta=171.6,167.8,162.2(\mathrm{~d}$, $J=246.3 \mathrm{~Hz}), 142.6,136.9,134.7,132.5(\mathrm{~d}, J=7.8 \mathrm{~Hz}), 131.3(\mathrm{~d}$, $J=3.2 \mathrm{~Hz}), 130.6,130.5,128.9,127.9,127.7,127.4,126.8,126.3$, $125.1,115.3(\mathrm{~d}, J=21.1 \mathrm{~Hz}), 104.6,101.7,65.4,64.2,61.4,40.6$, 13.5.

HRMS (ESI): $m / z[\mathrm{M}+\mathrm{H}]^{+}$calcd for $\mathrm{C}_{29} \mathrm{H}_{26} \mathrm{FN}_{2} \mathrm{O}_{3}{ }^{+}: 469.1922$; found: 469.1914 .

\section{Compound 10h}

Yield: $21.2 \mathrm{mg}$ (35\%); white crystalline solid; $\mathrm{mp} 104.1-106.0{ }^{\circ} \mathrm{C}$. IR (film): 3065, 2963, 2930, 1729, 1668, 1636, 1601, 1572, 1494, $1475,1446,1407,1368,1338,1287,1260,1226,1096,1045,1016$, $936,906,865,757,699,663,635,562,462 \mathrm{~cm}^{-1}$.

${ }^{1} \mathrm{H}$ NMR $\left(300 \mathrm{MHz}, \mathrm{CDCl}_{3}\right): \delta=7.62-7.60(\mathrm{~m}, 1 \mathrm{H}), 7.47-7.41(\mathrm{~m}$, $1 \mathrm{H}), 7.40-7.21(\mathrm{~m}, 6 \mathrm{H}), 7.21-7.06(\mathrm{~m}, 4 \mathrm{H}), 6.85-6.83(\mathrm{~m}, 1 \mathrm{H})$, $6.08(\mathrm{~d}, J=1.5 \mathrm{~Hz}, 1 \mathrm{H}), 5.82(\mathrm{~d}, J=7.8 \mathrm{~Hz}, 1 \mathrm{H}), 5.27(\mathrm{~d}$, $J=7.8 \mathrm{~Hz}, 1 \mathrm{H}), 4.94(\mathrm{~d}, J=1.5 \mathrm{~Hz}, 2 \mathrm{H}), 3.97(\mathrm{~d}, J=14.8 \mathrm{~Hz}$, $1 \mathrm{H}), 3.81-3.73(\mathrm{~m}, 1 \mathrm{H}), 3.68-3.59(\mathrm{~m}, 1 \mathrm{H}), 3.42(\mathrm{~d}, J=14.8 \mathrm{~Hz}$, $1 \mathrm{H}), 0.91(\mathrm{t}, J=7.1 \mathrm{~Hz}, 3 \mathrm{H})$.

${ }^{13} \mathrm{C} \mathrm{NMR}\left(75 \mathrm{MHz}, \mathrm{CDCl}_{3}\right): \delta=171.6,167.1,143.4,136.5,135.6$, $134.7,133.5,131.8,130.6,130.4,129.8,128.74,128.69,127.9$, 127.6, 127.5, 127.13, 127.09, 126.4, 124.9, 104.4, 100.9, 66.1, 64.1, $61.5,37.8,13.4$.

HRMS (ESI): $m / z[\mathrm{M}+\mathrm{H}]^{+}$calcd for $\mathrm{C}_{29} \mathrm{H}_{26} \mathrm{ClN}_{2} \mathrm{O}_{3}{ }^{+}: 485.1626$; found: 485.1641 .

\section{Compound 10i}

Yield: $26 \mathrm{mg}$ (43\%); white crystalline solid; $\mathrm{mp} 152.5-153.4{ }^{\circ} \mathrm{C}$.

IR (film): 3064, 3024, 2981, 2931, 1728, 1667, 1634, 1598, 1572, $1493,1476,1446,1408,1368,1339,1286,1226,1202,1120,1096$, $1047,1027,940,907,865,769,699,666,645 \mathrm{~cm}^{-1}$.

${ }^{1} \mathrm{H}$ NMR $\left(300 \mathrm{MHz}, \mathrm{CDCl}_{3}\right): \delta=7.46-7.08(\mathrm{~m}, 12 \mathrm{H}), 6.92-6.85$ $(\mathrm{m}, 1 \mathrm{H}), 6.10(\mathrm{~d}, J=1.2 \mathrm{~Hz}, 1 \mathrm{H}), 5.93(\mathrm{~d}, J=7.8 \mathrm{~Hz}, 1 \mathrm{H}), 5.31$ $(\mathrm{d}, J=7.8 \mathrm{~Hz}, 1 \mathrm{H}), 4.89(\mathrm{~d}, J=1.3 \mathrm{~Hz}, 1 \mathrm{H}), 4.77(\mathrm{~s}, 1 \mathrm{H}), 3.86$ $3.61(\mathrm{~m}, 2 \mathrm{H}), 3.52(\mathrm{~d}, J=14.3 \mathrm{~Hz}, 1 \mathrm{H}), 3.27(\mathrm{~d}, J=14.3 \mathrm{~Hz}, 1 \mathrm{H})$, $0.94(\mathrm{t}, J=7.1 \mathrm{~Hz}, 3 \mathrm{H})$.
${ }^{13} \mathrm{C}$ NMR $\left(75 \mathrm{MHz}, \mathrm{CDCl}_{3}\right): \delta=171.4,167.8,142.5,137.7,136.9$, 134.7, 134.3, 131.0, 130.6, 130.5, 129.7, 129.1, 129.0, 127.9, 127.7, 127.6, 127.3, 126.8, 126.4, 125.1, 104.6, 101.9, 65.3, 64.6, 61.5, 41.1, 13.5.

HRMS (ESI): $m / z[\mathrm{M}+\mathrm{H}]^{+}$calcd for $\mathrm{C}_{29} \mathrm{H}_{26} \mathrm{ClN}_{2} \mathrm{O}_{3}{ }^{+}: 485.1626$; found: 485.1613 .

\section{Compound 101}

Yield: $29.5 \mathrm{mg}$ (45\%); white crystalline solid; mp 162.0-164.0 ${ }^{\circ} \mathrm{C}$. IR (film): 2978, 1727, 1665, 1634, 1599, 1567, 1493, 1474, 1446, 1407, 1367, 1339, 1286, 1226, 1201, 1096, 1073, 1045, 939, 863, $783,751,698,668 \mathrm{~cm}^{-1}$.

${ }^{1} \mathrm{H}$ NMR $\left(300 \mathrm{MHz}, \mathrm{CDCl}_{3}\right): \delta=7.57(\mathrm{~s}, 1 \mathrm{H}), 7.44-7.26(\mathrm{~m}, 7 \mathrm{H})$, 7.24-7.08 (m, $4 \mathrm{H}), 6.91-6.84(\mathrm{~m}, 1 \mathrm{H}), 6.09(\mathrm{~d}, J=1.4 \mathrm{~Hz}, 1 \mathrm{H})$, $5.93(\mathrm{~d}, J=7.8 \mathrm{~Hz}, 1 \mathrm{H}), 5.31(\mathrm{~d}, J=7.8 \mathrm{~Hz}, 1 \mathrm{H}), 4.89(\mathrm{~d}$, $J=1.5 \mathrm{~Hz}, 1 \mathrm{H}), 4.76(\mathrm{~s}, 1 \mathrm{H}), 3.90-3.60(\mathrm{~m}, 2 \mathrm{H}), 3.52(\mathrm{~d}$, $J=14.2 \mathrm{~Hz}, 1 \mathrm{H}), 3.25(\mathrm{~d}, J=14.3 \mathrm{~Hz}, 1 \mathrm{H}), 0.94(\mathrm{t}, J=7.2 \mathrm{~Hz}$, $3 \mathrm{H})$.

${ }^{13} \mathrm{C}$ NMR $\left(75 \mathrm{MHz}, \mathrm{CDCl}_{3}\right): \delta=171.3,167.8,142.5,138.0,136.9$, $134.7,133.8,130.58,130.52,130.46,130.0,129.6,128.9,127.9$, $127.7,127.3,126.8,126.4,125.0,122.5,104.6,101.9,65.3,64.6$, $61.5,41.1,13.4$.

HRMS (ESI): $m / z[\mathrm{M}+\mathrm{H}]^{+}$calcd for $\mathrm{C}_{29} \mathrm{H}_{26} \mathrm{BrN}_{2} \mathrm{O}_{3}^{+}:$529.1121; found: 529.1126 .

\section{Compound 10m}

Yield: $30.2 \mathrm{mg}$ (54\%); white solid.

IR (film): 3063, 2982, 2937, 2905, 1732, 1666, 1633, 1602, 1579, 1492, 1447, 1409, 1370, 1343, 1261, 1189, 1097, 1071, 1026, 940, $910,865,773,754,701,667,646 \mathrm{~cm}^{-1}$.

${ }^{1} \mathrm{H}$ NMR (75 MHz, $\left.\mathrm{CDCl}_{3}\right): \delta=7.70-7.68(\mathrm{~m}, 2 \mathrm{H}), 7.46-7.30(\mathrm{~m}$, $3 \mathrm{H}), 7.25-7.06(\mathrm{~m}, 3 \mathrm{H}), 6.90(\mathrm{~d}, J=7.3 \mathrm{~Hz}, 1 \mathrm{H}), 6.14(\mathrm{~d}$, $J=1.6 \mathrm{~Hz}, 1 \mathrm{H}), 5.95(\mathrm{t}, J=8.1 \mathrm{~Hz}, 1 \mathrm{H}), 5.28(\mathrm{~d}, J=7.9 \mathrm{~Hz}, 1 \mathrm{H})$, $5.28(\mathrm{~d}, J=7.9 \mathrm{~Hz}, 1 \mathrm{H}), 5.21(\mathrm{~s}, 1 \mathrm{H}), 4.24-4.06(\mathrm{~m}, 2 \mathrm{H}), 3.84-$ $3.62(\mathrm{~m}, 2 \mathrm{H}), 3.22-2.91(\mathrm{~m}, 2 \mathrm{H}), 1.26(\mathrm{td}, J=7.1,2.6 \mathrm{~Hz}, 3 \mathrm{H})$, $0.91(\mathrm{t}, J=7.2 \mathrm{~Hz}, 3 \mathrm{H})$.

${ }^{13} \mathrm{C} \mathrm{NMR}\left(75 \mathrm{MHz}, \mathrm{CDCl}_{3}\right): \delta=170.8,169.9,167.9,142.5,137.3$, 135.1, 130.6, 130.4, 129.1, 128.1, 127.9, 127.1, 126.9, 126.3, 125.0, $104.3,98.8,65.2,61.5,61.1,61.0,39.0,14.1,13.4$.

HRMS (ESI): $m / z[\mathrm{M}+\mathrm{H}]^{+}$calcd for $\mathrm{C}_{26} \mathrm{H}_{27} \mathrm{~N}_{2} \mathrm{O}_{5}{ }^{+}: 447.1914$; found: 447.1917.

\section{Compound 11}

Yield: $16.9 \mathrm{mg}$ (38\%); white solid.

IR (film): 2963, 1733, 1665, 1631, 1525, 1492, 1447, 1369, 1346, $1261,1177,1088,1027,930,864,798,758,700,657 \mathrm{~cm}^{-1}$.

${ }^{1} \mathrm{H}$ NMR $\left(300 \mathrm{MHz}, \mathrm{CDCl}_{3}\right): \delta=7.79-7.63(\mathrm{~m}, 2 \mathrm{H}), 7.50-7.31(\mathrm{~m}$, $3 \mathrm{H}), 7.24-7.06(\mathrm{~m}, 3 \mathrm{H}), 6.96(\mathrm{~d}, J=7.5 \mathrm{~Hz}, 1 \mathrm{H}), 6.00-5.95(\mathrm{~m}$, $1 \mathrm{H}), 5.91(\mathrm{~d}, J=7.4 \mathrm{~Hz}, 1 \mathrm{H}), 5.54(\mathrm{~d}, J=7.8 \mathrm{~Hz}, 1 \mathrm{H}), 5.08-5.01$ $(\mathrm{m}, 1 \mathrm{H}), 4.95(\mathrm{~d}, J=10.5 \mathrm{~Hz}, 1 \mathrm{H}), 4.42-4.12(\mathrm{~m}, 3 \mathrm{H}), 1.32(\mathrm{t}$, $J=7.1 \mathrm{~Hz}, 3 \mathrm{H})$.

${ }^{13} \mathrm{C} \mathrm{NMR}\left(75 \mathrm{MHz}, \mathrm{CDCl}_{3}\right): \delta=169.0,167.8,139.9,137.4,134.7$, $130.7,129.4,129.0,128.4,127.9,127.0,126.9,125.2,107.2,97.4$, $62.5,61.7,54.4,14.2$.

HRMS (ESI): $m / z[\mathrm{M}+\mathrm{H}]^{+}$calcd for $\mathrm{C}_{22} \mathrm{H}_{21} \mathrm{~N}_{2} \mathrm{O}_{3}{ }^{+}: 361.1547$; found: 361.1539 .

\section{Compound 12}

Yield: $14.1 \mathrm{mg}$ (31\%); white solid.

IR (film): 3064, 2962, 2929, 2870, 1734, 1703, 1664, 1631, 1601, $1577,1524,1492,1452,1408,1371,1336,1276,1261,1210,1195$, $1178,1162,1087,1028,968,936,899,866,797,762,700,665$, $644,632 \mathrm{~cm}^{-1}$. 
${ }^{1} \mathrm{H}$ NMR $\left(300 \mathrm{MHz}, \mathrm{CDCl}_{3}\right): \delta=7.76-7.63(\mathrm{~m}, 2 \mathrm{H}), 7.46-7.32(\mathrm{~m}$, $3 \mathrm{H}), 7.23-7.08(\mathrm{~m}, 3 \mathrm{H}), 6.90(\mathrm{~d}, J=6.8 \mathrm{~Hz}, 1 \mathrm{H}), 6.13(\mathrm{~s}, 1 \mathrm{H})$, $5.94(\mathrm{~d}, J=7.9 \mathrm{~Hz}, 1 \mathrm{H}), 5.32(\mathrm{~d}, J=7.9 \mathrm{~Hz}, 1 \mathrm{H}), 5.10(\mathrm{~d}$, $J=7.8 \mathrm{~Hz}, 1 \mathrm{H}), 5.02-4.98(\mathrm{~m}, 1 \mathrm{H}), 4.03-3.88(\mathrm{~m}, 1 \mathrm{H}), 3.86-$ $3.56(\mathrm{~m}, 2 \mathrm{H}), 0.82(\mathrm{t}, J=7.1 \mathrm{~Hz}, 3 \mathrm{H})$.

${ }^{13} \mathrm{C} \mathrm{NMR}\left(75 \mathrm{MHz}, \mathrm{CDCl}_{3}\right): \delta=170.3,167.7,139.1,136.7,134.8$, $130.7,130.4,128.9,128.3,128.0,127.7,127.1,126.4,125.1,105.0$, 99.5, 62.0, 61.1, 57.7, 13.4 .

HRMS (ESI): $m / z[\mathrm{M}+\mathrm{H}]^{+}$calcd for $\mathrm{C}_{22} \mathrm{H}_{21} \mathrm{~N}_{2} \mathrm{O}_{3}{ }^{+}: 361.1547$; found: 361.1542 .

\section{Compound 14}

Yield: $31.3 \mathrm{mg}$ (47\%); white solid.

IR (film): 2979, 1729, 1671, 1523, 1486, 1449, 1381, 1355, 1278, $1225,1086,1018,959,802,758,702 \mathrm{~cm}^{-1}$.

${ }^{1} \mathrm{H}$ NMR $\left(300 \mathrm{MHz}, \mathrm{DMSO}-d_{6}\right): \delta=7.50-7.07(\mathrm{~m}, 14 \mathrm{H}), 6.26(\mathrm{~s}$, $1 \mathrm{H}), 6.08(\mathrm{~d}, J=1.6 \mathrm{~Hz}, 1 \mathrm{H}), 5.00(\mathrm{~d}, J=1.6 \mathrm{~Hz}, 1 \mathrm{H}), 4.85$ (s, $1 \mathrm{H}), 3.85(\mathrm{dq}, J=10.8,7.2 \mathrm{~Hz}, 1 \mathrm{H}), 3.67(\mathrm{dq}, J=10.8,7.1 \mathrm{~Hz}$, $1 \mathrm{H}), 3.52(\mathrm{~d}, J=14.4 \mathrm{~Hz}, 1 \mathrm{H}), 3.36(\mathrm{~d}, J=14.4 \mathrm{~Hz}, 1 \mathrm{H}), 0.98(\mathrm{t}$, $J=7.2 \mathrm{~Hz}, 3 \mathrm{H})$.

${ }^{13} \mathrm{C}$ NMR $\left(75 \mathrm{MHz}, \mathrm{CDCl}_{3}\right): \delta=171.4,167.6,142.8,137.5,135.3$, $134.5,130.9,130.5,129.6,129.1,128.6,127.9,127.7,127.6$, $127.53,127.48,126.5,125.2,101.3,98.5,65.5,63.5,61.7,41.4$, 13.6.

HRMS (ESI): $m / z[\mathrm{M}+\mathrm{H}]^{+}$calcd for $\mathrm{C}_{29} \mathrm{H}_{26} \mathrm{BrN}_{2} \mathrm{O}_{3}{ }^{+}: 529.1121$; found: 529.1113 .

\section{Compound 15}

Yield: $13.6 \mathrm{mg}$ (21\%); white solid.

IR (film): 3063, 2965, 1706, 1603, 1522, 1451, 1367, 1323, 1277, $1261,1198,1165,1108,1086,1024,861,799,756,711 \mathrm{~cm}^{-1}$.

${ }^{1} \mathrm{H}$ NMR $\left(300 \mathrm{MHz}, \mathrm{DMSO}-d_{6}\right): \delta=7.72-7.62(\mathrm{~m}, 2 \mathrm{H}), 7.50-7.24$ $(\mathrm{m}, 5 \mathrm{H}), 7.22-7.05(\mathrm{~m}, 5 \mathrm{H}), 7.02-6.90(\mathrm{~m}, 2 \mathrm{H}), 6.48(\mathrm{~s}, 1 \mathrm{H})$, $5.90(\mathrm{~d}, J=1.4 \mathrm{~Hz}, 1 \mathrm{H}), 5.35(\mathrm{~s}, 1 \mathrm{H}), 4.41(\mathrm{~d}, J=1.5 \mathrm{~Hz}, 1 \mathrm{H})$, 4.37-4.22 (m, $2 \mathrm{H}), 3.16(\mathrm{~s}, 2 \mathrm{H}), 1.33(\mathrm{t}, J=7.1 \mathrm{~Hz}, 3 \mathrm{H})$.

${ }^{13} \mathrm{C} \mathrm{NMR}\left(75 \mathrm{MHz}, \mathrm{CDCl}_{3}\right.$ ): $\delta=171.4,167.8,141.8,137.7,135.8$, $134.5,131.4,130.9,130.4,129.3,129.0,128.2,128.12,128.09$, $127.99,127.5,126.9,126.6,125.3,102.4,99.2,66.6,63.9,62.0$, 38.9, 14.0.

HRMS (ESI): $m / z[\mathrm{M}+\mathrm{H}]^{+}$calcd for $\mathrm{C}_{29} \mathrm{H}_{26} \mathrm{BrN}_{2} \mathrm{O}_{3}{ }^{+}: 529.1121$; found: 529.1099 .

\section{Acknowledgment}

This study was supported by the National Natural Science Foundation of China (No. 21172253), the Program for New Century Excellent Talents in University (No. NCET-11-0481), the National Scientific and Technology Supporting Program of China (No. 2011BAE06B05-5), the National S\&T Pillar Program of China (2012BAK25B03), the Scientific Research Foundation for the Returned Overseas Chinese Scholars, State Education Ministry, Chinese Universities Scientific Fund (No. 2012RC004), and Nutrichem Company.

Supporting Information for this article is available online at http://www.thieme-connect.com/ejournals/toc/synthesis.

\section{References}

(1) (a) Kartsev, V. G. Med. Chem. Res. 2004, 13, 325. (b) Madapa, S.; Tusi, Z.; Batra, S. Curr. Org. Chem. 2008, 12, 1116. (c) Kumar, S.; Bawa, S.; Gupta, H. Mini-Rev. Med. Chem. 2009, 9, 1648. (d) Musiol, R.; Serda, M.; Hensel-
Bielowka, S.; Polanski, J. Curr. Med. Chem. 2010, 17, 1960. (e) Kaur, K.; Jain, M.; Reddy, R. P.; Jain, R. Eur. J. Med. Chem. 2010, 45, 3245. (f) Liu, C. J.; Liu, D. Y.; Xiang, L. Acta Pharmacol. Sin. 2010, 45, 9. (g) Solomon, V. R.; Lee, H. Curr. Med. Chem. 2011, 18, 1488.

(2) For recent reviews on Et743, see: (a) Blay, J.-Y. Eur. J. Clin. Med. Oncol. 2010, 2, 1. (b) Vincenzi, B.; Napolitano, A.; Frezza, A. M.; Schiavon, G.; Santini, D.; Tonini, G. Pharmacogenomics J. 2010, 11, 865. (c) Carter, N. J.; Keam, S. J. Drugs 2010, 70, 355. (d) Ganjoo, K. N.; Patel, S. R. Expert Opin. Pharmacother. 2009, 10, 2735. (e) Boudou, L.; Baconnier, M.; Blay, J.-Y.; Lombard-Bohas, C.; Cassier, P. A. Expert Rev. Anticancer Ther. 2009, 9, 727. (f) Cuevas, C.; Francesch, A. Nat. Prod. Rep. 2009, 26, 322.

(3) Hisamine: (a) Pyo, M. K.; Lee, D. H.; Kim, D. H.; Lee, J. H.; Moon, J. C.; Chang, K. C.; Yun-Choi, H. S. Bioorg. Med. Chem. Lett. 2008, 18, 4110. (b) Pyo, M. K.; Kim, J. M.; Jin, J. L.; Chang, K. C.; Lee, D. H.; Yun-Choi, H. S. Thromb. Res. 2007, 120, 81.

(4) RIR-4-95: (a) Skogvall, S.; Dalence-Guzman, M. F.; Berglund, M.; Svensson, K.; Mesic, A.; Jonsson, P.; Persson, C. G. A.; Sterner, O. Pulm. Pharmacol. Ther. 2008, 21, 125. (b) Berglund, M.; Dalence-Guzman, M. F.; Skogvall, S.; Sterner, O. Bioorg. Med. Chem. 2008, 16, 2513. (c) Berglund, M.; Dalence-Guzman, M. F.; Skogvall, S.; Sterner, O. Bioorg. Med. Chem. 2008, 16, 2529.

(d) Dalence-Guzman, M. F.; Berglund, M.; Skogvall, S.; Sterner, O. Bioorg. Med. Chem. 2008, 16, 2499.

(5) Su, D.-S.; Lim, J. J.; Tinney, E.; Wan, B.-L.; Young, M. B.; Anderson, K. D.; Rudd, D.; Munshi, V.; Bahnck, C.; Felock, P. J.; Lu, M.; Lai, M.-T.; Touch, S.; Moyer, G.; DiStefano, D. J.; Flynn, J. A.; Liang, Y.; Sanchez, R.; Prasad, S.; Yan, Y.; Perlow-Poehnelt, R.; Torrent, M.; Miller, M.; Vacca, J. P.; Williams, T. M.; Anthony, N. J. Bioorg. Med. Chem. Lett. 2009, 19, 5119.

(6) Ramesh, E.; Manian, R. D. R. S.; Raghunathan, R.; Sainath, S.; Raghunathan, M. Bioorg. Med. Chem. 2009, 17, 660.

(7) Synthesis: (a) Scott, J. D.; Williams, R. M. Chem. Rev. 2002, 102, 1669. (b) Chrzanowska, M.; Rozwadowska, M. D. Chem. Rev. 2004, 104, 3341. (c) Siengalewicz, P.; Rinner, U.; Mulzer, J. Chem. Soc. Rev. 2008, 37, 2676. (d) Pulka, K. Curr. Opin. Drug Discovery Dev. 2010, 13, 669.

(e) Sridharan, V.; Suryavanshi, P. A.; Menéndez, J. C. Chem. Rev. 2011, 111, 7157. (f) Montalban, A. G. In Heterocycles in Natural Product Synthesis; Majumdar, K. C.; Chattopadhyay, S. K., Eds.; Wiley-VCH: Weinheim, 2011, 299-339. (g) Alajarin, R.; Burgos, C. In Modern Heterocyclic Chemistry; Vol. 3; Alvarez-Builla, J.; Vaquero, J. J.; Barluenga, J., Eds.; Wiley-VCH: Weinheim, 2011, 1527-1629. (h) Mekheimer, R. A.; Ahmed, E. A.; Sadek, K. U. Tetrahedron 2012, 68, 1637.

(8) For a review, see: Schantl, J. G. In Science of Synthesis; Vol. 27; Padwa, A., Ed.; Georg Thieme Verlag: Stuttgart, New York, 2004, 731-824.

(9) For examples of the cycloaddition of azomethine imines, see: (a) Stanovnik, B. Tetrahedron 1991, 47, 2925. (b) Žličar, M.; Stanovnik, B.; Tišler, M. Tetrahedron 1992, 48, 7965. (c) Svete, J.; Prešeren, A.; Stanovnik, B.; Golič, L.; Golič-Grdadolnik, S. J. Heterocycl. Chem. 1997, 34, 1323. (d) Roussi, F.; Bonin, M.; Chiaroni, A.; Micouin, L.; Riche, C.; Husson, H.-P. Tetrahedron Lett. 1999, 40, 3727. (e) Roussi, F.; Chauveau, A.; Bonin, M.; Micouin, L.; Husson, H.-P. Synthesis 2000, 1170. (f) Chuang, T.-H.; Sharpless, K. B. Helv. Chim. Acta 2000, 83, 1734. (g) Chauveau, A.; Martens, T.; Bonin, M.; Micouin, L.; Husson, H.-P. Synthesis 2002, 1885. (h) Panfil, I.; Urbanczyk-Lipkowska, Z.; Suwinska, K.; Solecka, J.; 
Chmielewski, M. Tetrahedron 2002, 58, 1199. (i) Belanger, G.; Hong, F.-T.; Overman, L. E.; Rogers, B. N.; Tellew, J.

E.; Trenkle, W. C. J. Org. Chem. 2002, 67, 7880.

(j) Shintani, R.; Fu, G. C. J. Am. Chem. Soc. 2003, 125, 10778. (k) Chung, F.; Chauveau, A.; Seltki, M.; Bonin, M.; Micouin, L. Tetrahedron Lett. 2004, 45, 3127. (1) Pezdirc, L.; Jovanovski, V.; Bevk, D.; Jakše, R.; Pirc, S.; Meden, A.; Stanovnik, B.; Svete, J. Tetrahedron 2005, 61, 3977.

(m) Suárez, A.; Downey, C. W.; Fu, G. C. J. Am. Chem. Soc. 2005, 127, 11244. (n) Shintani, R.; Hayashi, T. J. Am. Chem. Soc. 2006, 128, 6330. (o) Chen, W.; Yuan, X.-H.; Li, R.; Du, W.; Wu, Y.; Ding, L.-S.; Chen, Y.-C. Adv. Synth. Catal. 2006, 348, 1818. (p) Gergely, J.; Morgan, J. B.; Overman, L. E. J. Org. Chem. 2006, 71, 9144. (q) Chan, A.; Scheidt, K. A. J. Am. Chem. Soc. 2007, 129, 5334. (r) Suga, H.; Funyu, A.; Kakehi, A. Org. Lett. 2007, 9, 97. (s) Pezdirc, L.; Cerkovnik, J.; Pirc, S.; Stanovnik, B.; Svete, J. Tetrahedron 2007, 63, 991. (t) Svete, J. ARKIVOC 2006, (vii), 35. (u) Pezdirc, L.; Grošelj, U.; Meden, A.; Stanovnik, B.; Svete, J. J. Comb. Chem. 2007, 9, 717. (v) Shintani, R.; Murakami, M.; Hayashi, T. J. Am. Chem. Soc. 2007, 129 , 12356. (w) Chen, W.; Du, W.; Duan, Y.-Z.; Wu, Y.; Yang, S.-Y.; Chen, Y.-C. Angew. Chem. Int. Ed. 2007, 46, 7667. (x) Sibi, M. P.; Rane, D.; Stanley, L. M.; Soeta, T. Org. Lett. 2008, 10, 2971. (y) Kato, T.; Fujinami, S.; Ukaji, Y.; Inomata, K. Chem. Lett. 2008, 37, 342. (z) Perreault, C.; Goudreau, S. R.; Zimmer, L. E.; Charette, A. B. Org. Lett. 2008, 10, 689. (aa) Shapiro, N. D.; Shi, Y.; Toste, F. D. J. Am. Chem. Soc. 2009, 131, 11654. (ab) Keller, M.; Sido, A. S. S.; Pale, P.; Sommer, J. Chem.-Eur. J. 2009, 15, 2810 (ac) Hashimoto, T.; Maeda, Y.; Omote, M.; Nakatsu, H.; Maruoka, K. J. Am. Chem. Soc. 2010, 132, 4076. (ad) Ogawa, S.; Nishimine, T.; Tokunaga, E.; Shibata, N. Synthesis 2010, 3274. (ae) Hashimoto, T.; Omote, M.; Maruoka, K. Angew. Chem. Int. Ed. 2011, 50, 3489. (af) Hashimoto, T.; Omote, M.; Maruoka, K. Angew. Chem. Int. Ed. 2011, 50, 8952. (ag) Novak, A.; Bezenšek, J.;
Pezdirc, L.; Grošelj, U.; Kasunič, M.; Podlipnik, C.; Stanovnik, B.; Šimůnek, P.; Svete, J. Tetrahedron 2011, 67, 9729.

(10) Tamura, Y.; Miki, Y.; Nishikawa, Y.; Ikeda, M. J. Heterocycl. Chem. 1976, 13, 317.

(11) (a) Na, R.; Jing, C.; Xu, Q.; Jiang, H.; Wu, X.; Shi, J.; Zhong, J.; Wang, M.; Benitez, D.; Tkatchouk, E.; Goddard, W. A. III; Guo, H.; Kwon, O. J. Am. Chem. Soc. 2011, 133, 13337. (b) Jing, C.; Na, R.; Wang, B.; Liu, H.; Zhang, L.; Liu, J.; Wang, M.; Zhong, J.; Kwon, O.; Guo, H. Adv. Synth. Catal. 2012, 354, 1023. (c) Na, R.; Liu, H.; Li, Z.; Wang, B.; Liu, J.; Wang, M.-A.; Wang, M.; Zhong, J.; Guo, H. Tetrahedron 2012, 68, 2349. (d) Liu, J.; Liu, H.; Na, R.; Wang, G.; Wang, M.; Zhong, J.; Guo, H. Chem. Lett. 2012, $41,218$.

(12) For reviews, see: (a) Eicher, T.; Hauptmann, S. The Chemistry of Heterocycles, 2nd ed.; Wiley-VCH:

Weinheim, 2003. (b) Varvounis, G.; Fiamegos, Y.; Pilidis, G. Adv. Heterocycl. Chem. 2001, 80, 75. (c) Elguero, J. Pyrazoles: Comprehensive Heterocyclic Chemistry II; Vol. 3; Katritzky, A. R.; Rees, C. W.; Scriven, E. F. V., Eds.; Elsevier: Oxford, 1996, 1-75, and references cited therein (d) Marchand-Brynaert, J.; Ghosez, L. In Recent Progress in the Chemical Synthesis of Antibiotics; Lukacs, G.; Ohno, M., Eds.; Springer: Berlin, 1990. (e) Hanessian, S.;

McNaughton-Smith, G.; Lombart, H.-G.; Lubell, W. D. Tetrahedron 1997, 53, 12789. (f) Konaklieva, M. I.; Plotkin, B. J. Curr. Med. Chem. Anti-infect. Agents 2003, 2, 287.

(13) Legault, C.; Charette, A. B. J. Org. Chem. 2003, 68, 7119.

(14) Crystallographic data for $\mathbf{6 a}$ have been deposited with the Cambridge Crystallographic Data Centre as supplementary publication number CCDC 899345. These data can be obtained free of charge via www.ccdc.cam.ac.uk/data request/cif, or by emailing data_request@ccdc.cam.ac.uk, or by contacting The Cambridge Crystallographic Data Centre, 12, Union Road, Cambridge CB2 1EZ, UK; fax: +44(1223)336033. 